\title{
Improved energy efficiency within the Swedish steel industry: the importance of energy management and networking
}

\author{
Maria T. Johansson
}

Linköping University Post Print

Tweet

N.B.: When citing this work, cite the original article.

The original publication is available at www.springerlink.com:

Maria T. Johansson , Improved energy efficiency within the Swedish steel industry: the importance of energy management and networking, 2014, Energy Efficiency.

http://dx.doi.org/10.1007/s12053-014-9317-z

Copyright: Springer Verlag (Germany)

http://www.springerlink.com/?MUD=MP

Postprint available at: Linköping University Electronic Press

http://urn.kb.se/resolve?urn=urn:nbn:se:liu:diva-105873 


\title{
Improved energy efficiency within the Swedish steel industry - the importance of energy management and networking
}

\author{
Maria T. Johansson ${ }^{\mathrm{a}, \mathrm{b}^{*}}$ \\ ${ }^{a}$ Department of Management and Engineering, Division of Energy Systems, Linköping University, SE-581 83 \\ Linköping, Sweden \\ ${ }^{b}$ Department of Technology and Built Environment, Division of Energy and Mechanical Engineering, University \\ of Gävle, SE-801 76 Gävle, Sweden
}

* Corresponding author: fax: +46 (0)13 281788; E-mail address: maria.johansson@liu.se

\begin{abstract}
The iron and steel industry is an energy-intensive industry that consumes a significant portion of fossil fuel and electricity production. Climate change, the threat of an unsecure energy supply, and rising energy prices have emphasized the issue of improved energy efficiency in the iron and steel industry. However, an energy efficiency gap is well recognised, i.e. cost efficient measures are not implemented in practice. This study will go deeper into why this gap occurs by investigating energy management practices at 11 iron and steel companies in Sweden. Energy managers at the steel plants were interviewed about how they perceived their own and their companies' efforts to improve energy efficiency and how networking among energy managers influenced the efforts to improve energy efficiency. Reported barriers to improved energy efficiency were, for example, too long payback period, lack of profitability, lack of personnel, risk of production disruption, lack of time, and lack of commitment. Only three out of the eleven companies had assigned a person to work full time with energy management, and some of the energy managers were frustrated with not having enough time to work with energy issues. Generally, the respondents felt that they had support from senior management and that energy issues were prioritised, but only a few of the companies had made great efforts to involve employees in improving energy efficiency. Networking among Swedish steel companies was administered by the Swedish Steel Producers' Association, and their networking meetings contributed to the exchange of knowledge and ideas. In conclusion, Swedish steel companies regard improved energy efficiency as important but have much work left to do in this area. For example, vast amounts of excess heat are not being recovered and more efforts could be put into engaging employees and introducing a culture of energy efficiency.
\end{abstract}

\section{Keywords}

Energy efficiency, Energy management, Networking, Iron and steel industry, Interviews

\section{Introduction}

Improved energy efficiency has become an important issue for modern society. The European Commission has set a 2020 target date for reducing annual use of primary energy by $20 \%$ compared to amount used in 2005 (European Commission Eurostat 2013), and the Swedish government has set a 2020 target date to improve energy efficiency by 20\% compared to the 2008 level (Swedish Energy Agency 2013b). The great attention that improved energy efficiency has attracted is partly due to that fact that fossil fuel consumption results in the accumulation of greenhouse gas emissions and 
global climate change. Other factors that stimulate reduced use of primary energy are an unsecure energy supply and rising energy prices. In 2011 in Sweden, industry accounted for 38\% of the total energy use and the iron and steel industry accounted for $14 \%$ of the industrial energy usage (Swedish Energy Agency 2013a). The iron and steel industry is an energy-intensive industry and the main energy carriers are coal, coke, and electricity. The high energy intensity and the large share of fossil fuels make the energy issue important for the iron and steel industry. The steel industry is exposed to international competition and energy-efficient steel production can be a competitive advantage.

Improving energy efficiency is one way for process industries to reduce costs, and energy-saving technologies can be attractive from a business point of view. Examples of measures for improved energy efficiency are more efficient technologies, energy recovery in the manufacturing process, increased energy conversion efficiency, and optimisation of operational practices (Bunse et al. 2011).

Best available technologies (BAT) for the iron and steel industry have been improved and developed and operate close to their thermodynamic limits (European Commission 2010). Therefore, modern steel plants would have the largest energy efficiency potentials in support processes, energy recovery measures and optimisation of operational practices.

Firms often reject investments in measures for improved energy efficiency despite a positive net present value. This situation is referred to as the energy efficiency paradox or the energy efficiency gap (see, for example, Backlund et al. (2012); Jaffe and Stavins (1994); Martin et al. (2012)). This study will go deeper into why the energy efficiency gap occurs by investigating how energy managers reason in regard to energy use and improved energy efficiency. This is important in understanding their behaviour and actions in practice.

The aim of this paper is to investigate how energy managers in the Swedish iron and steel industry reason about factors that are proven to be important for successful energy management and to gain a deeper understanding of why energy efficiency measures are implemented or why they are not implemented. The objective is to capture different aspects and approaches to energy management, and the analysis focuses on how the energy managers perceive their own and their companies' efforts to improve energy efficiency. Five research questions guide this study's analysis:

1. Which barriers to improved energy efficiency has the respondent experienced?

2. Are responsibility and authority with regard to energy management delegated to the people in charge of energy issues at the company?

3. In what way is improved energy efficiency prioritised at the steel plant?

4. How are employees encouraged to engage in improved energy efficiency?

5. How does networking among energy managers influence the efforts to improve energy efficiency at Swedish steel plants?

\section{Theoretical foundation}

This study is based on the hypothesis that improved energy efficiency is a result of successful energy management, and this section begins with a description of the process of continuous improvements of industrial energy systems. However, there are both barriers to and driving forces for successful energy management, and it is important to be aware of these factors to achieve optimised industrial energy systems. These barriers and driving forces are presented in the last part of this section. 


\subsection{Energy management in industry}

Miles (2012) described management as the process of achieving an organisation's mission, strategies, goals, and objectives through the use of human resources, financial resources, physical resources, and informational resources. Energy management is the practice by which a company works strategically with energy issues, and an energy management system is a tool to be used by the company in order to implement energy management. Requirements for energy management systems are specified in the International Standard ISO 50001. The ISO 50001 follows the Plan-DoCheck-Act process for continuous improvements of the energy management system. The Plan-DoCheck-Act cycle ${ }^{1}$ was promoted by Deming (2000) as a management method for the control and continuous improvement of processes and products. Successful energy management must be strategically handled, and one key element is to have an organised and on-going programme of energy saving projects (Caffall 1995). The cycle in Figure 1 illustrates the process of successful energy management and can be described as:

1. Energy policy: the company's energy policy and vision are the foundation for priorities and decision-making with regard to energy issues

2. Energy planning: an energy review is conducted and targets, objectives, and action plans are established to meet legal requirements and the company's energy policy

3. Implementation and operation: the action plan is implemented and this involves, for example, procurement, operational control, communication, documentation, training, and awareness

4. Checking and correction: involves actions such as energy monitoring and energy performance analysis, reporting of results, evaluation of compliance with legal requirements and the company's energy policy, and corrective and preventive actions

5. Management review: opportunities to improve energy performance are identified and top management revises the company's energy policy, goals, and targets based on information and data extracted from the checking process. The review ensures continual improvements in energy performance and the energy management system

\footnotetext{
${ }^{1}$ Deming named the cycle the Shewhart cycle.
} 


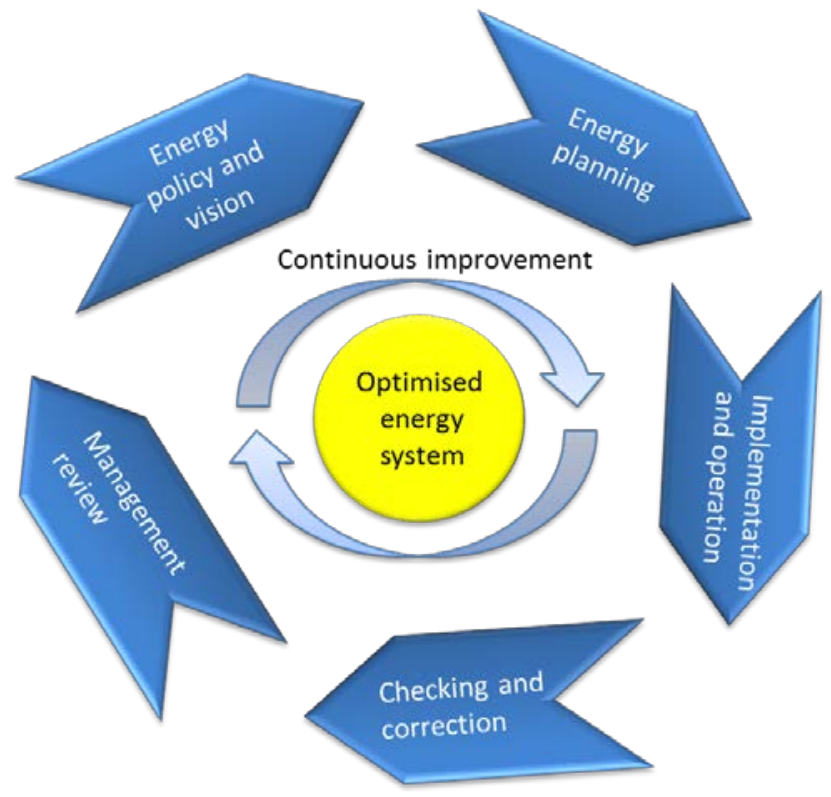

Figure 1. A cycle for continuous improvement of energy management and energy efficiency based on the Plan-Do-Check-Act approach. (Inspired by Deming (2000), International Organization for Standardization (2011), and the US Department of Energy (2013).)

The reliability of the framework for structured energy management was confirmed by Caffall (1995) and Abdelaziz et al. (2011). Caffall (1995) stated that successful energy management needs a strategic approach that includes 1) an initial energy audit; 2) senior management support; 3) monitoring of energy use; 4) recognition that management is as important as technology; and 5) an organised and on-going programme of energy-saving projects. Abdelaziz et al. (2011) identified the following four main components that are important for an effective energy management system: 1 ) analysis of historical data; 2 ) energy audits; 3 ) engineering analysis and investment proposals based on feasibility studies; and 4) personnel training and information.

The company's energy policy should give the energy manager the authority to be part of business planning, the purchasing of production and measuring equipment, energy reporting, and training of employees (Mashburn 2005). Planning is vital for any energy management programme, and to develop a successful plan the people assigned to implement the plan should participate in the planning process.

Successful energy management often relies on one ambitious person who makes things happen (Apeaning and Thollander 2013; Cagno and Trianni 2013; Rohdin et al. 2007; Thollander and Ottosson 2008). However, in order to have productive and permanent energy management it is important to develop an organisational structure that involves all employees. Employees are a great untapped resource in energy management programmes, and their ideas for improved energy efficiency should be solicited. Mashburn (2005) recommends that energy managers devote $20 \%$ of their working time to talking to employees. Jørgensen et al. (2008) concluded that human resource management results in sustainable and enhanced organisational performance through improved opportunities for learning, knowledge sharing, flexibility, commitment, creativity, and teamwork. The awareness that human resources are important has increased. An example of this is the development 
of the Danish energy management model that previously focused mainly on technical monitoring and measuring but now also includes information exchanges, communication, internal and external audits, and employee engagement (Christoffersen et al. 2006). The energy manager should have support from an energy team with representatives from accounting or purchasing, production, and maintenance and one person from every other major department (Mashburn 2005). The purpose of this team should be to bring new ideas and to supplement skills that the energy manager lacks.

Compliance with legal requirements should be evaluated, and energy management practices should be continuously evaluated and corrected. This requires monitoring and measuring energy use through sub-metering and recurring internal energy audits. Mashburn (2005) advises keeping reporting requirements as simple as possible (e.g., visualising energy utilisation in a continuous graph and comparing it to a baseline year). However, data and key performance indicators often do not allow for effective energy performance evaluation and decision support (Sivill et al. 2012). In addition, industry often experiences problems in finding software suitable for visualising key energy efficiency performance indicators and simulation tools that can integrate energy efficiency performance (Bunse et al. 2011).

In every energy management programme it is important to have support from the senior management (Caffall 1995; Mashburn 2005). Moreover, the energy manager and the energy committee should revise the energy policy annually and recommend updating when necessary (Mashburn 2005). Previous research (Martin et al. 2012) has found that if climate change issues are administrated by an environmental manager or an energy manager the firms have more climatefriendly practices. Moreover, if there was a hierarchical proximity between the environmental manager and the CEO the firm had a more climate-friendly management. However, if the CEO was in charge of climate-change issues the reverse was true.

\subsection{Barriers to and driving forces for improved industrial energy efficiency}

Climate-friendly management practices have been associated with higher productivity and better energy efficiency (Bloom et al. 2010; Martin et al. 2012), and investments in energy-saving technology have been shown to not only save energy but also benefit productivity (Pye and McKane 2000; Worrell et al. 2003; Bloom et al. 2010). However, many companies have not integrated these practices in their operations. Thollander and Ottosson (2010) found in their survey of energy management practices in the Swedish pulp and paper industry and the foundry industry that only $40 \%$ of the pulp and paper mills and $25 \%$ of the foundries could be considered successful in terms of energy management. However, the degree of adoption of energy management practices increased with the intensity of energy use. According to a survey by Christoffersen et al. (2006), 14\% of manufacturing industries in Denmark practiced energy management and Ates and Durakbasa (2012) reported that $22 \%$ of Turkish energy-intensive industries practiced corporate energy management.

Barriers to improved energy efficiency have been intensively studied (see e.g. (Apeaning and Thollander 2013; De Groot et al. 2001; Rohdin and Thollander 2006; Rohdin et al. 2007; Sardianou 2008; Sorrell 2000; Trianni et al. 2013a; Trianni et al. 2013b; Trianni et al. 2013c; Walsh and Thornley 2012; Fleiter et al. 2011)). Sorrell (2000) classified barriers to energy efficiency into the following three categories: economic (which can be further divided into market failure and non-market failure), behavioural, and organisational. Palm and Thollander (2010) analysed and discussed these categories further, and Cagno et al. (2013) and Sudhakara Reddy (2013) proposed novel taxonomies 
of barriers that could be used in empirical studies. The former study categorised the internal barriers into technology-related barriers, information barriers, economic barriers, behavioural barriers, organisational barriers, and barriers related to competences and awareness. The external barriers were categorised into markets, governmental politics, technology and services suppliers, designers and manufacturers, energy suppliers, and capital suppliers. The study by Sudhakara Reddy (2013) associated barriers and drivers to the institutions that create it and the institutions that are influenced by it. The barriers were classified as micro (obstacles that are unique to a particular project), meso (obstacles related to the organization working with a project), and macro (barriers at state, market and civil society level). Fleiter et al. (2011) reviewed the current status of bottom-up models for industrial energy demand, and they analysed how these models considered barriers to the adoption of new technologies. Empirical studies on barriers to improved industrial energy efficiency have been conducted in Ghana (Apeaning and Thollander 2013), Greece (Sardianou 2008), Sweden (Rohdin and Thollander 2006; Rohdin et al. 2007; Thollander et al. 2007; Thollander and Ottosson 2008; Trianni et al. 2013a; Brunke et al. 2014), Italy (Trianni et al. 2013a; Trianni et al. 2013b; Trianni et al. 2013c), Germany (Trianni et al. 2013a), the UK (Walsh and Thornley 2012), Belgium ((Venmans 2014), and the Netherlands (De Groot et al. 2001). Table 1 presents barriers to improved energy efficiency that were identified in these previous studies. The barriers seem to be quite similar in different countries and industry sectors even if their relative importance is different.

Table 1. Overview of barriers to energy efficiency measures investigated in previous research. A star before a reference shows that the barrier was ranked as one of the three most important barriers in that study. The barrier may have its origin within the firm (I) or outside the firm (E). The categories are based on the taxonomy proposed by Cagno et al. (2013) for empirical investigations.

\begin{tabular}{|c|c|c|c|}
\hline Category & Barrier & Sector & Key references \\
\hline \multirow[t]{10}{*}{$\begin{array}{l}\text { Technology- } \\
\text { related barriers }\end{array}$} & $\begin{array}{l}\text { Technology is } \\
\text { inappropriate at } \\
\text { this site }(E)\end{array}$ & $\begin{array}{l}\text { Steel and aluminum, food processing, } \\
\text { plastic products, petrochemicals and } \\
\text { chemicals, cement, textile, paper (Ghana) }\end{array}$ & $\begin{array}{l}\text { Apeaning and } \\
\text { Thollander (2013) }\end{array}$ \\
\hline & & $\begin{array}{l}\text { Iron and steel (Japan, USA, Australia, South } \\
\text { Korea, China, India and Canada) }\end{array}$ & $\begin{array}{l}\text { *Okazaki and } \\
\text { Yamaguchi (2011) }\end{array}$ \\
\hline & & $\begin{array}{l}\text { Non-energy intensive manufacturing } \\
\text { (Sweden) }\end{array}$ & $\begin{array}{l}\text { Rohdin and Thollander } \\
\text { (2006) }\end{array}$ \\
\hline & & Foundry (Sweden) & Rohdin et al. (2007) \\
\hline & & Pulp and paper (Sweden) & $\begin{array}{l}\text { *Thollander and } \\
\text { Ottosson (2008) }\end{array}$ \\
\hline & & $\begin{array}{l}\text { Foundry (Finland, France, Germany, Italy, } \\
\text { Poland, Spain, Sweden) }\end{array}$ & Trianni et al. (2013a) \\
\hline & & Manufacturing SMEs (Northern Italy) & Trianni et al. (2013c) \\
\hline & & SMEs (Germany) & Fleiter et al. (2012) \\
\hline & & $\begin{array}{l}\text { Primary metal manufacturing SMEs } \\
\text { (Northern Italy) }\end{array}$ & Trianni et al. (2013b) \\
\hline & $\begin{array}{l}\text { Technology can } \\
\text { only be } \\
\text { implemented after }\end{array}$ & $\begin{array}{l}\text { Chemical, basic metals, metal products, } \\
\text { horticulture, food, paper (Netherlands) }\end{array}$ & *De Groot et al. (2001) \\
\hline
\end{tabular}


existing technology

has been replaced

(I)

Iron and steel (Japan, USA, Australia, South

Manufacturing SMEs (Northern Italy)

Technologies not available (E)

Implementation
cancelled due to
change in operation
(I)

Primary metal manufacturing SMEs

(Northern Italy)

Iron and steel (Sweden)

SMEs (Germany)

Brunke et al. (2014)

Fleiter et al. (2012)

Chemical, basic metals, metal products, horticulture, food, paper (Netherlands) not sufficiently important (I)

Department or workers not accountable for energy costs (I)

Long decision chains (I)

Lack of time or other priorities (I) (2011) Poland, Spain, Sweden)

Steel (Korea)

Lee (2014)

Thollander and

Ottosson (2008)

Foundry (Finland, France, Germany, Italy, Poland, Spain, Sweden)

Steel and aluminum, food processing, plastic products, petrochemicals and

Apeaning and Thollander (2013)
Korea, China, India and Canada)

Okazaki and Yamaguchi

Trianni et al. (2013c)

Trianni et al. (2013b)

*De Groot et al. (2001)

Foundry (Finland, France, Germany, Italy, Trianni et al. (2013a) chemicals, cement, textile, paper (Ghana)

Non-energy intensive manufacturing

(Sweden)

Foundry (Sweden)

Manufacturing SMEs (Sweden)

Pulp and paper (Sweden)

Non-energy intensive manufacturing SMEs (Northern Italy)

Trianni et al. (2013a)

*Rohdin and

Thollander (2006)

Rohdin et al. (2007)

*Thollander et al. (2007)

Thollander and Ottosson (2008)

Trianni and Cagno (2012)

Foundry (Finland, France, Germany, Italy, Poland, Spain, Sweden)

Manufacturing SMEs (Northern Italy)

Trianni et al. (2013c)

SMEs (Germany)

Fleiter et al. (2012);

Primary metal manufacturing SMEs

(Northern Italy)

Steel (Korea)

Trianni et al. (2013b)

Lee (2014)

Iron and steel (Sweden) 


Low priority given
to energy
management (I)

Insufficient top

management

support (I)

Energy manager lacks influence (I)

Energy objectives not integrated into operating, maintenance, or purchasing procedures (I)

Lack of submetering (I)

Slim organisation (I)
Difficult to
implement due to
internal
organisation (I)
Possible poor
performance of
equipment (E)

Ceramic, cement, lime (Belgium)

Venmans (2014)

Chemical, basic metals, metal products, horticulture, food, paper (Netherlands)

Foundry (Sweden)

Manufacturing SMEs (Sweden)

Pulp and paper (Sweden)

Foundry (Finland, France, Germany, Italy, Poland, Spain, Sweden)

Steel (Korea)

Iron and steel (Sweden)

Pulp and paper (Sweden)

Foundry (Finland, France, Germany, Italy, Poland, Spain, Sweden)

Steel (Korea)

Iron and steel (Sweden)

Foundry (Finland, France, Germany, Italy, Poland, Spain, Sweden)

Steel (Korea)

Foundry (Sweden)

Manufacturing SMEs (Sweden)

Pulp and paper (Sweden)

Foundry (Finland, France, Germany, Italy, Poland, Spain, Sweden)

Manufacturing SMEs (Sweden)

Pulp and paper (Sweden)

Foundry (Finland, France, Germany, Italy, Poland, Spain, Sweden)

Chemical, basic metals, metal products, horticulture, food, paper (Netherlands)

Chemical, basic metals, metal products, horticulture, food, paper (Netherlands)

Thollander and
De Groot et al. (2001)

Rohdin et al. (2007)

Thollander et al. (2007)

Thollander and

Ottosson (2008)

Trianni et al. (2013a)

Lee (2014)

Brunke et al. (2014)

Thollander and Ottosson (2008)

Trianni et al. (2013a)

*Lee (2014)

Brunke et al. (2014)

Trianni et al. (2013a)

Lee (2014)

Rohdin et al. (2007)

Thollander et al. (2007)

Ottosson (2008)

Trianni et al. (2013a)

Thollander et al. (2007)

Thollander and

Ottosson (2008)

Trianni et al. (2013a)

De Groot et al. (2001)

De Groot et al. (2001)
Information equipment $(E)$ 
Non-energy intensive manufacturing

(Sweden)

Foundry (Sweden)

Pulp and paper (Sweden)

Foundry (Finland, France, Germany, Italy, Poland, Spain, Sweden)

Steel (Korea)

No good overview of existing technologies (I/E)

Poor information for the energy efficiency decision (I/E)

Insufficient technological information (E)

Technical feasibility was not studied before (I)

Difficulty in obtaining information on the energy use of purchased equipment $(E)$

Chemical, basic metals, metal products, horticulture, food, paper (Netherlands) (Northern Italy)

SMEs (Germany) Korea, China, India and Canada)

Ceramic, cement, lime (Belgium)

Non-energy intensive manufacturing (Sweden)
Information issues

on energy contracts

(E)

Information not
clear by technology
suppliers (E)

Trustworthiness of the information source (E)
Non-energy intensive manufacturing SMEs

*Trianni and Cagno (2012)

Fleiter et al. (2012)

Iron and steel (Japan, USA, Australia, South

*Rohdin and

Thollander (2006)

Rohdin et al. (2007)

Thollander et al. (2007)

Trianni et al. (2013a)

Lee (2014)

*Trianni et al. (2013c)

Primary metal manufacturing SMEs (Northern Italy)

Manufacturing SMEs (Northern Italy)

Primary Metal manufacturing SMEs (Northern Italy)
Manufacturing SMEs (Northern Italy)

Primary metal manufacturing SMEs
Rohdin and Thollander

Rohdin et al. (2007)

Thollander and

Trianni et al. (2013a)

Okazaki and Yamaguchi (2011)

*Venmans (2014)

Foundry (Finland, France, Germany, Italy, Poland, Spain, Sweden)

Manufacturing SMEs (Northern Italy)

*Trianni et al. (2013b)

Trianni et al. (2013c)

Trianni et al. (2013b)

Trianni et al. (2013c)

Trianni et al. (2013b) 


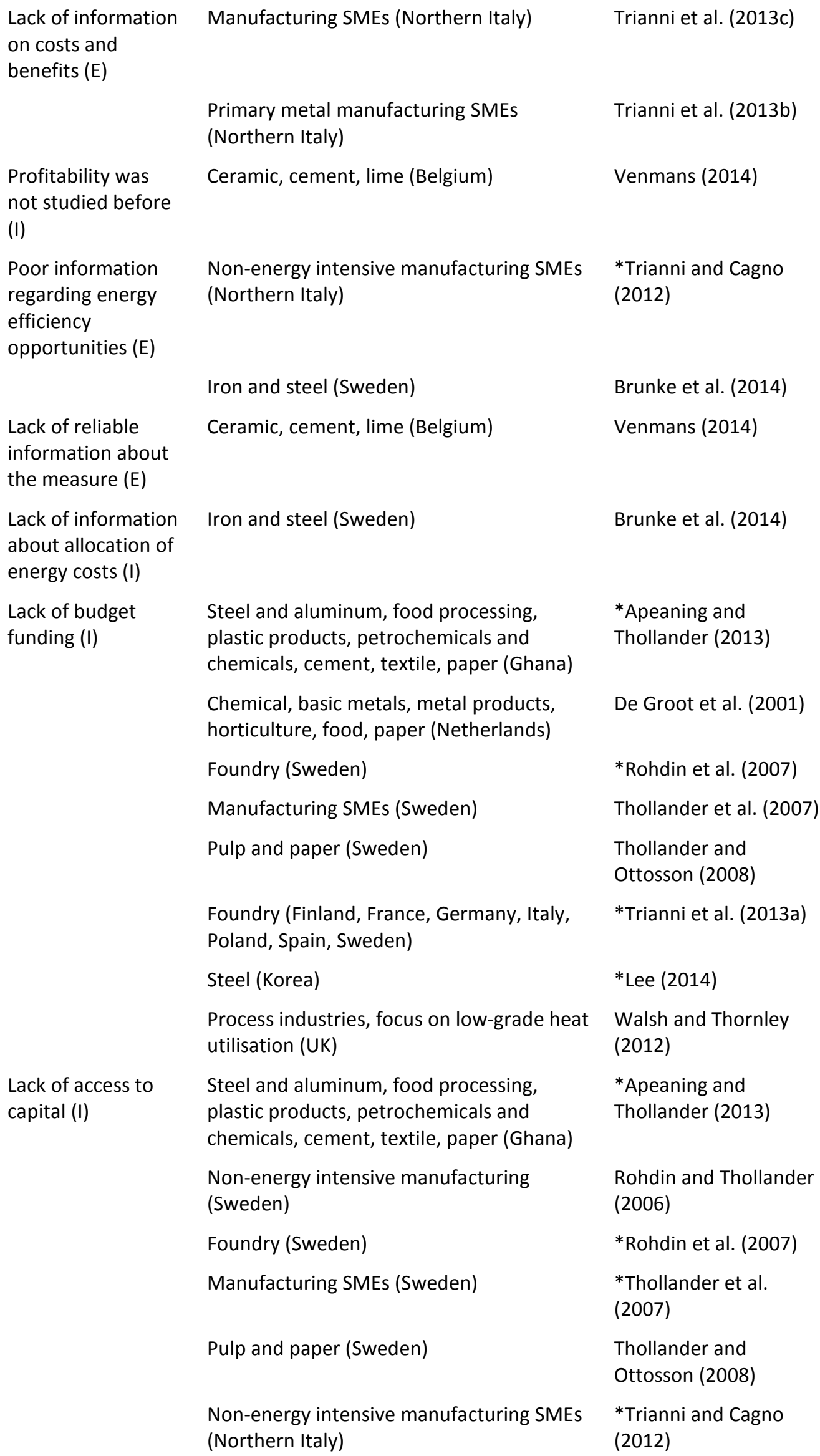


Foundry (Finland, France, Germany, Italy,

Poland, Spain, Sweden)

Manufacturing SMEs (Northern Italy)

Metals, machinery, food/drink, chemicals, paper, textiles (Greece)

Primary metal manufacturing SMEs

(Northern Italy)

Iron and steel (Sweden)

Ceramic, cement, lime (Belgium)

Problems with external financing (E)

Investment costs
(E)

High perceived costs of energysaving measures (I/E)

Measure not profitable (I/E) Intervention not sufficiently profitable (I/E)

Cost of production disruption/hassle/ inconvenience $(\mathrm{I} / \mathrm{E})$
Chemical, basic metals, metal products, horticulture, food, paper (Netherlands)

SMEs (Germany)

Ceramic, cement, lime (Belgium)

Manufacturing SMEs (Northern Italy)

Process industries, focus on low-grade heat utilisation (UK)

SMEs (Germany)

Primary metal manufacturing SMEs (Northern Italy)

Metals, machinery, food/drink, chemicals, paper, textiles (Greece)

SMEs (Germany)

Iron and steel (Japan, USA, Australia, South Korea, China, India and Canada)

Manufacturing SMEs (Northern Italy)

Metals, machinery, ffoodood/drink, chemicals, paper, textiles (Greece)

Primary metal manufacturing SMEs (Northern Italy)

Steel and aluminum, food processing, plastic products, petrochemicals and chemicals, cement, textile, paper (Ghana)

Iron and steel (Japan, USA, Australia, South Korea, China, India and Canada)

Non-energy intensive manufacturing (Sweden)

Foundry (Sweden)

Pulp and paper (Sweden)
Trianni et al. (2013a)

Trianni et al. (2013c)

*Sardianou (2008)

Trianni et al. (2013b)

Brunke et al. (2014)

Venmans (2014)

De Groot et al. (2001)

Fleiter et al. (2012)

Venmans (2014)

*Trianni et al. (2013c)

Walsh and Thornley (2012)

*Fleiter et al. (2012)

Trianni et al. (2013b)

*Sardianou (2008)

*Fleiter et al. (2012)

Okazaki and Yamaguchi (2011)

Trianni et al. (2013c)

Sardianou (2008)

Trianni et al. (2013b)

Apeaning and Thollander (2013)

Okazaki and Yamaguchi (2011)

*Rohdin and Thollander (2006)

Thollander et al. (2007)

*Thollander and

Ottosson (2008) 
Foundry (Finland, France, Germany, Italy,

Poland, Spain, Sweden)

Ceramic, cement, lime (Belgium)

Cost of identifying opportunities, analysing cost effectiveness, and tendering (I/E)

Cost of staff replacement/ retirement/ retraining $(\mathrm{I})$

Interventionrelated risks (I/E)

Do not want to implement a new technology because it requires replacing a relatively new facility (I)

Technical risks such as risk of production disruptions (I/E)
Steel and aluminum, food processing, plastic products, petrochemicals and chemicals, cement, textile, paper (Ghana)

Non-energy intensive manufacturing (Sweden)

Foundry (Sweden)

Manufacturing SMEs (Sweden)

Pulp and paper (Sweden)

Foundry (Finland, France, Germany, Italy, Poland, Spain, Sweden)

Steel (Korea)

Foundry (Finland, France, Germany, Italy, Poland, Spain, Sweden)

Manufacturing SMEs (Northern Italy)

Primary metal manufacturing SMEs (Northern Italy)

Iron and steel (Japan, USA, Australia, South Korea, China, India and Canada)

Steel and aluminum, food processing, plastic products, petrochemicals and chemicals, cement, textile, paper (Ghana)

Non-energy intensive manufacturing (Sweden)

Foundry (Sweden)

Manufacturing SMEs (Sweden)

Pulp and paper (Sweden)

Foundry (Finland, France, Germany, Italy, Poland, Spain, Sweden)

SMEs (Germany)

Steel (Korea)

Iron and Steel (Sweden)
Trianni et al. (2013a)

Venmans (2014)

Apeaning and

Thollander (2013)

Rohdin and Thollander (2006)

Rohdin et al. (2007)

Thollander et al. (2007)

Thollander and

Ottosson (2008)

Trianni et al. (2013a)

Lee (2014)

Trianni et al. (2013a)

Trianni et al. (2013c)

Trianni et al. (2013b)

Okazaki and Yamaguchi (2011)

Apeaning and

Thollander (2013)

Rohdin and Thollander (2006)

*Rohdin et al. (2007)

Thollander et al. (2007)

*Thollander and

Ottosson (2008)

Trianni et al. (2013a)

Fleiter et al. (2012)

*Lee (2014)

*Brunke et al. (2014) 


\begin{tabular}{|c|c|c|c|}
\hline & \multirow[t]{2}{*}{ External risks (E) } & Manufacturing SMEs (Northern Italy) & Trianni et al. (2013c) \\
\hline & & $\begin{array}{l}\text { Primary metal manufacturing SMEs } \\
\text { (Northern Italy) }\end{array}$ & Trianni et al. (2013b) \\
\hline & \multirow[t]{4}{*}{ Hidden costs (I/E) } & Manufacturing SMEs (Northern Italy) & *Trianni et al. (2013c) \\
\hline & & $\begin{array}{l}\text { Primary metal manufacturing SMEs } \\
\text { (Northern Italy) }\end{array}$ & *Trianni et al. (2013b) \\
\hline & & Iron and steel (Sweden) & Brunke et al. (2014) \\
\hline & & Ceramic, cement, lime (Belgium) & *Venmans (2014) \\
\hline \multirow[t]{4}{*}{ Market } & \multirow[t]{2}{*}{$\begin{array}{l}\text { Uncertainty about } \\
\text { future energy } \\
\text { prices (E) }\end{array}$} & SMEs (Germany) & Fleiter et al. (2012) \\
\hline & & Iron ans steel industry (Sweden) & Brunke et al. (2014) \\
\hline & $\begin{array}{l}\text { Uncertainty } \\
\text { regarding the } \\
\text { company's future } \\
\text { (E) }\end{array}$ & $\begin{array}{l}\text { Foundry (Finland, France, Germany, Italy, } \\
\text { Poland, Spain, Sweden) }\end{array}$ & Trianni et al. (2013a) \\
\hline & $\begin{array}{l}\text { Business/market } \\
\text { uncertainty }(E)\end{array}$ & Steel (Korea) & Lee (2014) \\
\hline \multirow[t]{11}{*}{ Behavioural } & \multirow[t]{11}{*}{$\begin{array}{l}\text { Other priorities for } \\
\text { capital investments } \\
\text { (I) }\end{array}$} & $\begin{array}{l}\text { Steel and aluminum, food processing, } \\
\text { plastic products, petrochemicals and } \\
\text { chemicals, cement, textile, paper (Ghana) }\end{array}$ & $\begin{array}{l}\text { *Apeaning and } \\
\text { Thollander (2013) }\end{array}$ \\
\hline & & $\begin{array}{l}\text { Iron and steel (Japan, USA, Australia, South } \\
\text { Korea, China, India and Canada) }\end{array}$ & $\begin{array}{l}\text { *Okazaki and } \\
\text { Yamaguchi (2011) }\end{array}$ \\
\hline & & $\begin{array}{l}\text { Chemical, basic metals, metal products, } \\
\text { horticulture, food, paper (Netherlands) }\end{array}$ & *De Groot et al. (2001) \\
\hline & & $\begin{array}{l}\text { Non-energy intensive manufacturing } \\
\text { (Sweden) }\end{array}$ & $\begin{array}{l}\text { Rohdin and Thollander } \\
\text { (2006) }\end{array}$ \\
\hline & & Foundry (Sweden) & ${ }^{*}$ Rohdin et al. (2007) \\
\hline & & Manufacturing SMEs (Sweden) & Thollander et al. (2007) \\
\hline & & Pulp and paper (Sweden) & $\begin{array}{l}\text { Thollander and } \\
\text { Ottosson (2008) }\end{array}$ \\
\hline & & $\begin{array}{l}\text { Non-energy intensive manufacturing SMEs } \\
\text { (Northern Italy) }\end{array}$ & $\begin{array}{l}\text { Trianni and Cagno } \\
(2012)\end{array}$ \\
\hline & & $\begin{array}{l}\text { Foundry (Finland, France, Germany, Italy, } \\
\text { Poland, Spain, Sweden) }\end{array}$ & *Trianni et al. (2013a) \\
\hline & & $\begin{array}{l}\text { Metals, machinery, food/drink, chemicals, } \\
\text { paper, textiles (Greece) }\end{array}$ & Sardianou (2008) \\
\hline & & SMEs (Germany) & *Fleiter et al. (2012) \\
\hline
\end{tabular}


Primary metal manufacturing SMEs

(Northern Italy)

Steel (Korea)

Iron ans steel (Sweden)

Ceramic, cement, lime (Belgium)

Conflicts of interest Steel and aluminum, food processing, within the company plastic products, petrochemicals and (I)

Imperfect

evaluation criteria (I)

Lack of interest in energy efficiency interventions $(\mathrm{I} / \mathrm{E})$

Barriers related to competence Poland, Spain, Sweden)

SMEs (Germany)

Manufacturing SMEs (Northern Italy)

Primary metal manufacturing SMEs

(Northern Italy)

Manufacturing SMEs (Northern Italy)

Primary metal manufacturing SMEs

(Northern Italy)

Inertia (I)

Manufacturing SMEs (Northern Italy)

Primary metal manufacturing SMEs

(Northern Italy)

(Northern Italy)
Implementing the interventions (I)

Lack of technical skills (I/E) chemicals, cement, textile, paper (Ghana)

Foundry (Finland, France, Germany, Italy,

Non-energy intensive manufacturing SMEs

Manufacturing SMEs (Northern Italy)

SMEs (Germany)

Primary metal manufacturing SMEs (Northern Italy)

Steel and aluminum, food processing, plastic products, petrochemicals and chemicals, cement, textile, paper (Ghana) Iron and steel (Japan, USA, Australia, South Korea, China, India and Canada)

Non-energy intensive manufacturing (Sweden)

Manufacturing SMEs (Sweden)

Pulp and paper (Sweden)

Non-energy intensive manufacturing SMEs (Northern Italy)

Foundry (Finland, France, Germany, Italy, Poland, Spain, Sweden)

Steel (Korea)
Trianni et al. (2013b)

Lee (2014)

*Brunke et al. (2014)

*Venmans (2014)

Apeaning and

Thollander (2013)

Trianni et al. (2013a)

Fleiter et al. (2012)

Trianni et al. (2013c)

Trianni et al. (2013b)

Trianni et al. (2013c)

*Trianni et al. (2013b)

Trianni et al. (2013c)

Trianni et al. (2013b)

Trianni and Cagno

(2012)

Trianni et al. (2013c)

Fleiter et al. (2012)

Trianni et al. (2013b)

Apeaning and

Thollander (2013)

Okazaki and Yamaguchi (2011)

Rohdin and Thollander (2006)

Thollander et al. (2007)

Thollander and Ottosson (2008)

Trianni and Cagno

Trianni et al. (2013a)

Lee (2014) 
Iron and steel (Sweden)

Difficulty in gathering external skills (E)

Identifying energy efficiency opportunities (I)

Identifying the inefficiencies (I)

$\begin{array}{ll}\text { Awareness } & \begin{array}{l}\text { Lack of staff } \\ \text { awareness (I) }\end{array} \\ & \\ \text { Government/ } & \\ \text { Bolitics } & \\ & \text { procedures to get } \\ \text { governmental } \\ \text { financial support } \\ \text { (E) } \\ \text { Inadequate } \\ \text { national policies } \\ \text { such as inefficient } \\ \text { national standards } \\ \text { and regulations (E) } \\ \text { Inadequate }\end{array}$

Non-energy intensive manufacturing SMEs

(Northern Italy)

Manufacturing SMEs (Northern Italy)

Primary metal manufacturing SMEs

(Northern Italy)

Non-energy intensive manufacturing

(Sweden)

Foundry (Sweden)

Pulp and paper (Sweden)

Non-energy intensive manufacturing SMEs (Northern Italy)

Foundry (Finland, France, Germany, Italy, Poland, Spain, Sweden)

Manufacturing SMEs (Northern Italy)

Primary metal manufacturing SMEs (Northern Italy)

Steel (Korea)

Manufacturing SMEs (Northern Italy)

Primary metal manufacturing SMEs

(Northern Italy)

Non-energy intensive manufacturing

(Sweden)

Manufacturing SMEs (Sweden)

Pulp and paper (Sweden)

Non-energy intensive manufacturing SMEs (Northern Italy)

Foundry (Finland, France, Germany, Italy, Poland, Spain, Sweden)

Steel (Korea)

Metals, machinery, food/drink, chemicals, paper, textiles (Greece)

Iron and steel (Japan, USA, Australia, South Korea, China, India and Canada)

Iron and steel (Japan, USA, Australia, South
*Okazaki and Yamaguchi (2011)

Brunke et al. (2014)

Trianni and Cagno

(2012)

Trianni et al. (2013c)

Trianni et al. (2013b)

Rohdin and Thollander (2006)

Rohdin et al. (2007)

Thollander and Ottosson (2008)

Trianni and Cagno (2012)

Trianni et al. (2013a)

Trianni et al. (2013b)

Trianni et al. (2013b)

Lee (2014)

Trianni et al. (2013c)

Trianni et al. (2013b)

Rohdin and Thollander (2006)

Thollander et al. (2007)

Thollander and Ottosson (2008)

Trianni and Cagno (2012)

Trianni et al. (2013a)

Lee (2014)

*Sardianou (2008)

Okazaki and Yamaguchi 
government

Okazaki and Yamaguchi (2011) protection of intellectual property (E)

When summarising the findings in the empirical studies on barriers to improved energy efficiency in the steel industry (Apeaning and Thollander 2013; Brunke et al. 2014; De Groot et al. 2001; Lee 2014; Okazaki and Yamaguchi 2011; Sardianou 2008), the most frequently high-ranked barriers were the economic barriers "lack of budget funding", "lack of access to capital" and "technical risks such as risk of production disruption", and the behavioural barrier "other priorities for capital investments". In conclusion, internal economic barriers were in majority among the highest-ranked barriers. Brunke et al. (2014) identified the four most important barriers to improved energy efficiency in the Swedish iron and steel industry as "technical risks such as risk of production disruption", "lack of access to capital", "other priorities for capital investments", and " "lack of time or other priorities".

Empirical studies of the driving forces for improved industrial energy efficiency have been published (Apeaning and Thollander 2013; Brunke et al. 2014; Christoffersen et al. 2006; Hasanbeigi et al. 2010; Rohdin and Thollander 2006; Rohdin et al. 2007; Thollander and Ottosson 2008; Cagno and Trianni 2013; Thollander et al. 2007; Thollander et al. 2013; Venmans 2014). The main driving forces studied in these publications are presented in Table 2 . The driving forces varied somewhat between the studies, e.g., the driving force improving working conditions was only found to be of major importance in the studies from Ghana and Thailand and not in the European studies. However, longterm energy strategy, commitment from top management, environmental company profile, cost reduction from lowered energy use, people with real ambition, and the threat of rising energy prices were considered important driving forces in the majority of the studies.

Table 2. Overview of driving forces for energy efficiency measures investigated in previous research. A star before a reference shows that the driving force was ranked as one of the three most important in that study. The driving force may have its origin within the firm (I) or outside the firm (E). The categories are based on the taxonomy proposed by Cagno et al. (2013) for empirical investigations.

\begin{tabular}{llll}
\hline Category & Driving force & Sector & Key references \\
\hline $\begin{array}{l}\text { Technology-related } \\
\text { drivers }\end{array}$ & New solutions (E) & $\begin{array}{l}\text { Manufacturing SMEs } \\
\text { (Italy) }\end{array}$ & Cagno and Trianni (2013) \\
& $\begin{array}{l}\text { Replace obsolete } \\
\text { equipment/production } \\
\text { expansion (I) }\end{array}$ & $\begin{array}{l}\text { Ceramic, cement, lime } \\
\text { (Belgium) }\end{array}$ & Venmans (2014) \\
& $\begin{array}{l}\text { Long-term energy strategy } \\
\text { Organisational }\end{array}$ & $\begin{array}{l}\text { Steel and aluminum, } \\
\text { food processing, } \\
\text { plastic products, }\end{array}$ & $\begin{array}{l}\text { Apeaning and Thollander } \\
\text { (2013) }\end{array}$
\end{tabular}




\begin{tabular}{|c|c|c|}
\hline & $\begin{array}{l}\text { petrochemicals and } \\
\text { chemicals, cement, } \\
\text { textile, paper (Ghana) }\end{array}$ & \\
\hline & Cement (Thailand) & Hasanbeigi et al. (2010) \\
\hline & Textile (Thailand) & Hasanbeigi et al. (2010) \\
\hline & $\begin{array}{l}\text { Non-energy intensive } \\
\text { manufacturing } \\
\text { (Sweden) }\end{array}$ & $\begin{array}{l}{ }^{*} \text { Rhodin and Thollander } \\
(2006)\end{array}$ \\
\hline & Foundry (Sweden) & ${ }^{*}$ Rohdin et al. (2007) \\
\hline & $\begin{array}{l}\text { Manufacturing SMEs } \\
\text { (Sweden) }\end{array}$ & *Thollander et al. (2007) \\
\hline & $\begin{array}{l}\text { Foundry (Finland, } \\
\text { France, Germany, } \\
\text { Italy, Poland, Spain, } \\
\text { Sweden) }\end{array}$ & Thollander et al. (2013) \\
\hline & $\begin{array}{l}\text { Pulp and paper } \\
\text { (Sweden) }\end{array}$ & $\begin{array}{l}\text { *Thollander and Ottosson } \\
(2008)\end{array}$ \\
\hline & $\begin{array}{l}\text { Iron and steel } \\
\text { (Sweden) }\end{array}$ & *Brunke et al. (2014) \\
\hline $\begin{array}{l}\text { Commitment from top } \\
\text { management (I) }\end{array}$ & $\begin{array}{l}\text { Foundry (Finland, } \\
\text { France, Germany, } \\
\text { Italy, Poland, Spain, } \\
\text { Sweden) }\end{array}$ & ${ }^{*}$ Thollander et al. (2013) \\
\hline & $\begin{array}{l}\text { Iron and steel } \\
\text { (Sweden) }\end{array}$ & *Brunke et al. (2014) \\
\hline & $\begin{array}{l}\text { Ceramic, cement, lime } \\
\text { (Belgium) }\end{array}$ & *Venmans (2014) \\
\hline $\begin{array}{l}\text { Network within the } \\
\text { company/group (I) }\end{array}$ & $\begin{array}{l}\text { Steel and aluminum, } \\
\text { food processing, } \\
\text { plastic products, } \\
\text { petrochemicals and } \\
\text { chemicals, cement, } \\
\text { textile, paper (Ghana) }\end{array}$ & $\begin{array}{l}\text { Apeaning and Thollander } \\
\text { (2013) }\end{array}$ \\
\hline & $\begin{array}{l}\text { Foundry (Finland, } \\
\text { France, Germany, } \\
\text { Italy, Poland, Spain, } \\
\text { Sweden) }\end{array}$ & Thollander et al. (2013) \\
\hline & $\begin{array}{l}\text { Pulp and paper } \\
\text { (Sweden) }\end{array}$ & $\begin{array}{l}\text { Thollander and Ottosson } \\
(2008)\end{array}$ \\
\hline $\begin{array}{l}\text { Environmental } \\
\text { management system (I) }\end{array}$ & $\begin{array}{l}\text { Steel and aluminum, } \\
\text { food processing, } \\
\text { plastic products, } \\
\text { petrochemicals and } \\
\text { chemicals, cement, } \\
\text { textile, paper (Ghana) }\end{array}$ & $\begin{array}{l}\text { Apeaning and Thollander } \\
\text { (2013) }\end{array}$ \\
\hline & Foundry (Sweden) & Rohdin et al. (2007) \\
\hline & Pulp and paper & Thollander and Ottosson \\
\hline
\end{tabular}




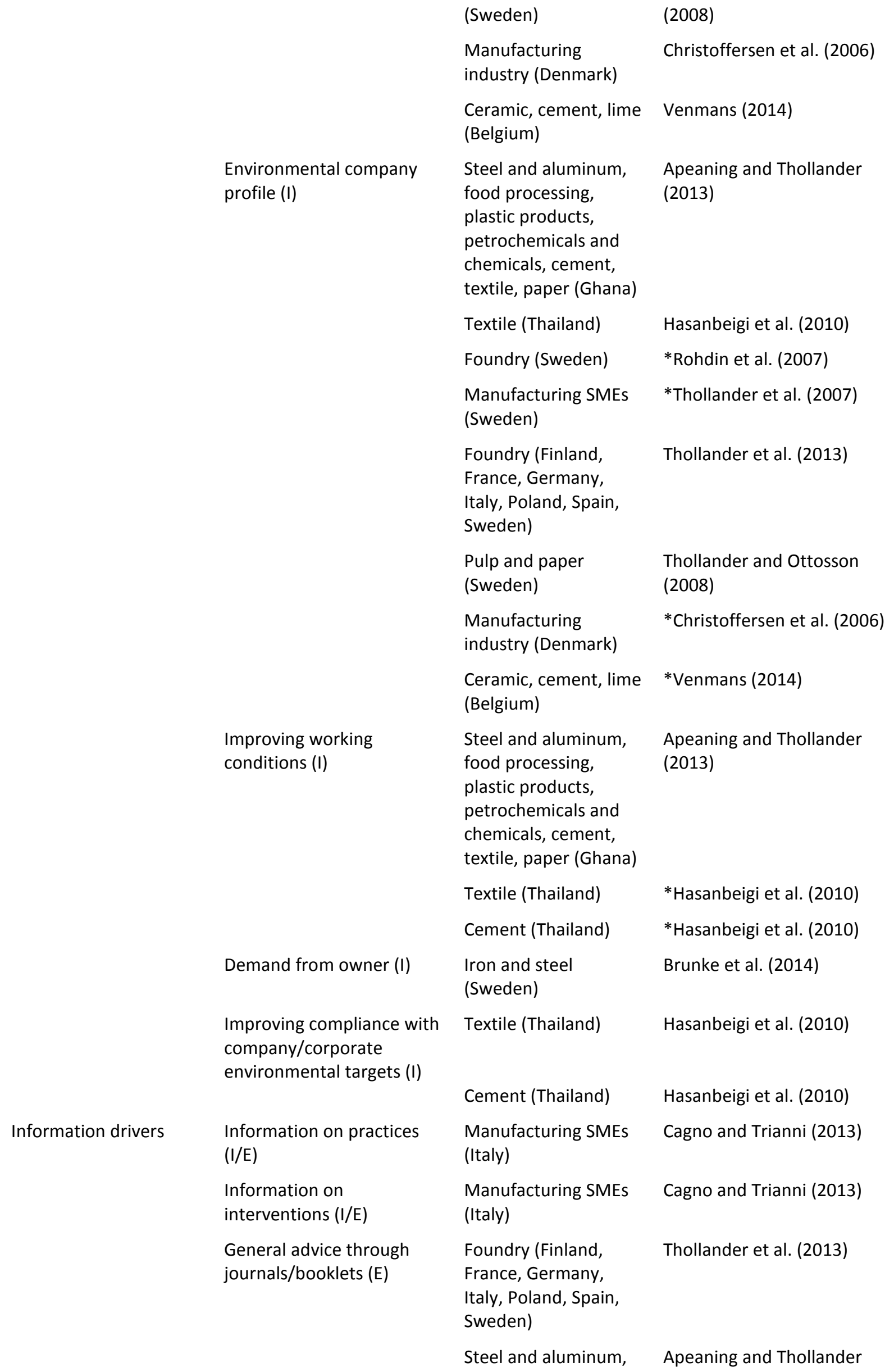


Market prices $(E)$

International competition (E)

Customer questions and demand (E)

External pressures (E)

Cost reduction from lowered energy use (I) food processing,

plastic products,

petrochemicals and

chemicals, cement, textile, paper (Ghana)

Result of in-house R\&D (I)

Ceramic, cement, lime (Belgium)

Steel and aluminum, food processing, plastic products, petrochemicals and chemicals, cement, textile, paper (Ghana)

Non-energy intensive manufacturing

(Sweden)

Foundry (Finland, France, Germany, Italy, Poland, Spain, Sweden)

Pulp and paper (Sweden)

Thollander and Ottosson (2008)

Ceramic, cement, lime (Belgium)

Iron and steel (Sweden)

*Rhodin and Thollander (2006)

*Thollander et al. (2013)

*Apeaning and Thollander (2013)

*Venmans (2014)

Brunke et al. (2014)

Foundry (Sweden)

Rohdin et al. (2007)

Foundry (Finland, France, Germany, Italy, Poland, Spain, Sweden)

Pulp and paper (Sweden)

Iron and steel (Sweden)

Manufacturing SMEs (Italy)

Foundry (Finland, France, Germany, Italy, Poland, Spain, Sweden)

Manufacturing industry (Denmark)

Thollander and Ottosson (2008)

Brunke et al. (2014)

Cagno and Trianni (2013)

Thollander et al. (2013)

Manufacturing SMEs (Italy)

Steel and aluminum, food processing, plastic products,
Christoffersen et al. (2006)

*Cagno and Trianni (2013)

*Apeaning and Thollander (2013) 


\begin{tabular}{|c|c|c|c|}
\hline & & $\begin{array}{l}\text { petrochemicals and } \\
\text { chemicals, cement, } \\
\text { textile, paper (Ghana) }\end{array}$ & \\
\hline & & $\begin{array}{l}\text { Pulp and paper } \\
\text { (Sweden) }\end{array}$ & $\begin{array}{l}\text { *Thollander and Ottosson } \\
\text { (2008) }\end{array}$ \\
\hline & & $\begin{array}{l}\text { Manufacturing } \\
\text { industry (Denmark) }\end{array}$ & ${ }^{*}$ Christoffersen et al. (2006) \\
\hline & & $\begin{array}{l}\text { Foundry (Finland, } \\
\text { France, Germany, } \\
\text { Italy, Poland, Spain, } \\
\text { Sweden) }\end{array}$ & *Thollander et al. (2013) \\
\hline & & $\begin{array}{l}\text { Iron and steel } \\
\text { (Sweden) }\end{array}$ & *Brunke et al. (2014) \\
\hline & Long-term benefits (E) & $\begin{array}{l}\text { Manufacturing SMEs } \\
\text { (Italy) }\end{array}$ & *Cagno and Trianni (2013) \\
\hline & $\begin{array}{l}\text { Improving product quality } \\
\text { (I) }\end{array}$ & Textile (Thailand) & *Hasanbeigi et al. (2010) \\
\hline & & Cement (Thailand) & *Hasanbeigi et al. (2010) \\
\hline & $\begin{array}{l}\text { Investment subsidies or } \\
\text { beneficial loans }(E)\end{array}$ & $\begin{array}{l}\text { Foundry (Finland, } \\
\text { France, Germany, } \\
\text { Italy, Poland, Spain, } \\
\text { Sweden) }\end{array}$ & Thollander et al. (2013) \\
\hline & $\begin{array}{l}\text { Allowances or public } \\
\text { financing }(E)\end{array}$ & $\begin{array}{l}\text { Manufacturing SMEs } \\
\text { (Italy) }\end{array}$ & *Cagno and Trianni (2013) \\
\hline & & $\begin{array}{l}\text { Pulp and paper } \\
\text { (Sweden) }\end{array}$ & $\begin{array}{l}\text { Thollander and Ottosson } \\
\text { (2008) }\end{array}$ \\
\hline & $\begin{array}{l}\text { Lower costs of } \\
\text { consultancies }(E)\end{array}$ & $\begin{array}{l}\text { Manufacturing SMEs } \\
\text { (Italy) }\end{array}$ & Cagno and Trianni (2013) \\
\hline & $\begin{array}{l}\text { Energy performance } \\
\text { contracts (E) }\end{array}$ & $\begin{array}{l}\text { Pulp and paper } \\
\text { (Sweden) }\end{array}$ & $\begin{array}{l}\text { Thollander and Ottosson } \\
\text { (2008) }\end{array}$ \\
\hline & $\begin{array}{l}\text { Support from sector } \\
\text { organisation }(E)\end{array}$ & $\begin{array}{l}\text { Iron and steel } \\
\text { (Sweden) }\end{array}$ & Brunke et al. (2014) \\
\hline & & $\begin{array}{l}\text { Manufacturing SMEs } \\
\text { (Italy) }\end{array}$ & Cagno and Trianni (2013) \\
\hline Behavioural & Management sensitivity (I) & $\begin{array}{l}\text { Manufacturing SMEs } \\
\text { (Italy) }\end{array}$ & Cagno and Trianni (2013) \\
\hline & $\begin{array}{l}\text { People with real ambition } \\
\text { (I) }\end{array}$ & $\begin{array}{l}\text { Steel and aluminum, } \\
\text { food processing, } \\
\text { plastic products, } \\
\text { petrochemicals and } \\
\text { chemicals, cement, } \\
\text { textile, paper (Ghana) }\end{array}$ & $\begin{array}{l}\text { Apeaning and Thollander } \\
\text { (2013) }\end{array}$ \\
\hline & & $\begin{array}{l}\text { Manufacturing } \\
\text { industry (Denmark) }\end{array}$ & Christoffersen et al. (2006) \\
\hline & & $\begin{array}{l}\text { Manufacturing SMEs } \\
\text { (Italy) }\end{array}$ & Cagno and Trianni (2013) \\
\hline & & $\begin{array}{l}\text { Non-energy intensive } \\
\text { manufacturing }\end{array}$ & $\begin{array}{l}\text { *Rhodin and Thollander } \\
\text { (2006) }\end{array}$ \\
\hline
\end{tabular}




\begin{tabular}{|c|c|c|c|}
\hline & & (Sweden) & \\
\hline & & Foundry (Sweden) & *Rohdin et al. (2007) \\
\hline & & $\begin{array}{l}\text { Manufacturing SMEs } \\
\text { (Sweden) }\end{array}$ & *Thollander et al. (2007) \\
\hline & & $\begin{array}{l}\text { Pulp and paper } \\
\text { (Sweden) }\end{array}$ & $\begin{array}{l}\text { *Thollander and Ottosson } \\
\text { (2008) }\end{array}$ \\
\hline & & $\begin{array}{l}\text { Foundry (Finland, } \\
\text { France, Germany, } \\
\text { Italy, Poland, Spain, } \\
\text { Sweden) }\end{array}$ & Thollander et al. (2013) \\
\hline & & $\begin{array}{l}\text { Iron and steel } \\
\text { (Sweden) }\end{array}$ & Brunke et al. (2014) \\
\hline $\begin{array}{l}\text { Drivers related to } \\
\text { competence }\end{array}$ & $\begin{array}{l}\text { Increase of internal } \\
\text { competences (I) }\end{array}$ & $\begin{array}{l}\text { Manufacturing SMEs } \\
\text { (Italy) }\end{array}$ & Cagno and Trianni (2013) \\
\hline & $\begin{array}{l}\text { Access to energy efficiency } \\
\text { experts (E) }\end{array}$ & $\begin{array}{l}\text { Manufacturing SMEs } \\
\text { (Italy) }\end{array}$ & Cagno and Trianni (2013) \\
\hline & & $\begin{array}{l}\text { Iron and steel } \\
\text { (Sweden) }\end{array}$ & Brunke et al. (2014) \\
\hline Government/politics & $\begin{array}{l}\text { Compliance with } \\
\text { regulatory issues }(E)\end{array}$ & $\begin{array}{l}\text { Steel and aluminum, } \\
\text { food processing, } \\
\text { plastic products, } \\
\text { petrochemicals and } \\
\text { chemicals, cement, } \\
\text { textile, paper (Ghana) }\end{array}$ & $\begin{array}{l}\text { *Apeaning and Thollander } \\
\text { (2013) }\end{array}$ \\
\hline & & $\begin{array}{l}\text { Manufacturing SMEs } \\
\text { (Italy) }\end{array}$ & Cagno and Trianni (2013) \\
\hline & & $\begin{array}{l}\text { Pulp and paper } \\
\text { (Sweden) }\end{array}$ & $\begin{array}{l}\text { Thollander and Ottosson } \\
\text { (2008) }\end{array}$ \\
\hline & & Cement (Thailand) & Hasanbeigi et al. (2010) \\
\hline & & $\begin{array}{l}\text { Iron and steel } \\
\text { (Sweden) }\end{array}$ & Brunke et al. (2014) \\
\hline & Voluntary agreements (E) & $\begin{array}{l}\text { Ceramic, cement, lime } \\
\text { (Belgium) }\end{array}$ & Venmans (2014) \\
\hline & & $\begin{array}{l}\text { Iron and steel } \\
\text { (Sweden) }\end{array}$ & Brunke et al. (2014) \\
\hline & $\begin{array}{l}\text { Energy and emissions taxes } \\
\text { (E) }\end{array}$ & $\begin{array}{l}\text { Foundry (Finland, } \\
\text { France, Germany, } \\
\text { Italy, Poland, Spain, } \\
\text { Sweden) }\end{array}$ & Thollander et al. (2013) \\
\hline & & $\begin{array}{l}\text { Steel and aluminum, } \\
\text { food processing, } \\
\text { plastic products, } \\
\text { petrochemicals and } \\
\text { chemicals, cement, } \\
\text { textile, paper (Ghana) }\end{array}$ & $\begin{array}{l}\text { Apeaning and Thollander } \\
\text { (2013) }\end{array}$ \\
\hline & & $\begin{array}{l}\text { Iron and steel } \\
\text { (Sweden) }\end{array}$ & Brunke et al. (2014) \\
\hline
\end{tabular}


The four most frequently high-ranked driving forces for improved energy efficiency in the steel industry (Apeaning and Thollander 2013; Brunke et al. 2014) were the economic driver "cost reduction from lowered energy use", the organisational drivers "long-term energy strategy" and "commitment from top management", and the market driver "threat of rising energy prices". All of these driving forces have their origin within the firm, except for the driving force "threat of rising energy prices". According to Brunke et al. (2014), the four most important driving forces for improved energy efficiency in the Swedish iron and steel industry were "commitment from top management", "cost reduction from lowered energy use", "long-term energy strategy", and "people with real ambitions".

\section{Swedish iron and steel industry}

In Sweden, there are two steel plants that produce steel from iron ore in the blast furnace - oxygen furnace (BF-BOF) process and ten plants that produce steel from scrap in electric arc furnaces (EAF). In addition, one plant produces iron powder from iron ore through a process called direct reduction (DRI plant). Figure 2 shows the locations of these steel plants. In addition, there are 16 steel processing plants with only rolling mills, wire drawing and tube mills. In 2011, the Swedish iron and steel industry had approximately 18,000 employees and produced 3.9 million tonnes of steel products. Almost 93\% of the steel products were exported to other countries (Jernkontoret 2014). Swedish iron and steel production resulted in an annual energy use of 21.2 TWh (Swedish Energy Agency 2012).

Six iron and steel companies in Sweden participated in the Swedish programme for improving energy efficiency in energy-intensive industry (PFE). Energy audits ${ }^{2}$ performed at these companies had identified 81 measures for improved energy efficiency, which corresponded to a total energy efficiency potential of $46.6 \mathrm{GWh} / \mathrm{y}$. Moreover, Brunke et al. (2014) studied the adoption of energy conservation measures in the Swedish iron and steel industry. Their study, which included nine steelproducing companies and 14 steel companies with only shaping processes, revealed an estimated energy efficiency potential of $7.3 \%$ of the total energy use in the studied companies if adopting costeffective energy conservation technologies. When adding the effects of sound energy management practices to the techno-economic potential, the energy efficiency potential was estimated at $9.7 \%$ of the total energy use. This equals approximately $1.8 \mathrm{TWh} / \mathrm{y}$. The companies participating in the study accounted for over $80 \%$ of the Swedish iron and steel industry's total turnover and the results were obtained by sending a questionnaire to the person in charge of energy issues at the company.

\footnotetext{
${ }^{2}$ The information was provided by the Swedish Energy Agency.
} 


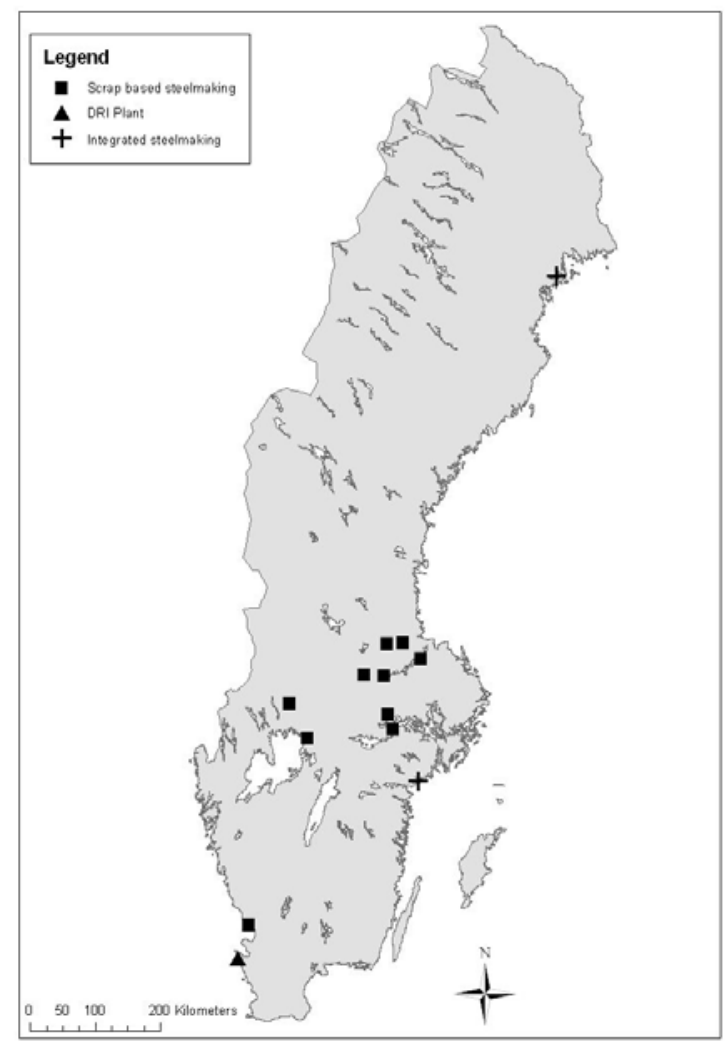

Figure 2. Localization of steel-producing plants in Sweden. Squares represents steel plants with steel production from scrap in electric arc furnaces, pluses are steel production from iron-ore in blast furnaces, and the triangle is steel production through direct reduction of iron ore.

Jernkontoret, the Swedish Steel Producers' Association, was founded in 1747. Jernkontoret is owned by Swedish Steel Works and its main function is to "safeguard the interests of the Swedish steel industry through working actively to ensure the best possible operation conditions for the industry in Sweden" (Jernkontoret 2014). Moreover, Jernkontoret strives to be a builder of both national and international networks. One of the national networks is the Energy NETwork that is designed to improve the efficiency of the steel industry energy system. The network is referred to as ENET-Steel in this paper. The network was founded in 2005 and the purpose of the network is to conduct activities that focus on energy consulting, education, the collection and dissemination of information, and the exchange of experiences. At the time for this study, ENET-Steel had 265 members. The network has a chat site on Jernkontoret's homepage where members can exchange information. There is an Energy Council at Jernkontoret with representatives from member companies. Subordinate to the Council is the Committee for Improved Energy Efficiency, and this committee is responsible for ENET-Steel. The committee organises network meetings twice a year that are held either in Stockholm at Jernkontoret's head office or at a member steel plant. These meetings have approximately 25-50 participants. Colleagues to the network members are also welcome to visit the meetings. Activities at the meetings are presentations on current legislations and policies with regard to energy use and $\mathrm{CO}_{2}$ emissions, presentations of new technologies, and presentations of "good examples" of improved energy efficiency. The presentations of implemented measures and their 
economy are also displayed in the e-book "Energy Handbook" on Jernkontoret's web-page and distributed by e-mail to all members. Examples of subjects that have been discussed the meetings are heat recovery in exhaust gas boilers and heat delivery to district heating networks, optimisation of pickling-bath parameters and routines to save energy, improved energy efficiency by simple and comprehensible work instructions, energy thinking in new investment decisions, electricity production from excess heat, improved control of compressor operation in order to produce more compressed air with existing equipment, and excess heat from furnaces replacing oil-boilers for heating.

\section{Methodology}

This study is explorative in nature and aims to answer questions like 'How', 'Why', and 'What'. Therefore, a qualitative research method is appropriate. In-depth interviews are a qualitative data collection method that can be used to elicit in-depth information from relatively few persons (Kvale 1996). Therefore, in-depth interviews are used to gain a deeper understanding of how the energy managers perceive their own and their companies' efforts to improve energy efficiency.

The in-depth interviews were conducted in the spring of 2012 with the members of the Energy Council at Jernkontoret. The persons interviewed were selected partly because of their membership on the Council. This was because one of the objectives of this study was to investigate the importance of networking between energy managers, and the Energy Council serves as a meeting place for energy managers at Swedish steel plants. The interviewees were representatives from 11 Swedish steel plants and they were responsible for energy issues at their respective companies. Their titles were full-time or part-time energy coordinators, environmental managers, or persons responsible for energy procurements at the company.

The in-depth interviews were semi-structured and the questions in the interview guide were asked in the order that seemed appropriate at each interview. Additional questions that correlated to the respondents' answers were asked in order to improve the clarity of the response. The interviews were completed face-to-face at the respondents' companies and the average interview lasted 60 minutes. To find organisational information about ENET-Steel an additional interview was conducted with the person responsible for organising ENET-Steel at Jernkontoret. All interviews were audiorecorded and transcribed in full and the respondents are anonymised in the paper.

The main themes of the interview guide were:

1. Background information (e.g. number of employees, production and energy use, the respondents' education and years of employment at the company)

2. Implemented, planned, and rejected measures for improved energy efficiency (e.g. types of measures, barriers to implementation)

3. Organisation of energy management (e.g. authority, decision-making, finance and investments, senior management support)

4. Prioritisation of energy issues (e.g. energy vision, resources to implement measures)

5. Motivation of employees (e.g. education programmes, information)

6. Networking (e.g. activities, advantages/disadvantages with membership, other energyrelated cooperations) 
Analysis of the interviews was conducted by identifying themes and categories that could answer the research questions. A coding scheme that defined the themes and categories was developed and used to identify specific sections of the interview data that were of interest for the study. By finding common themes and categories, it was possible to compile and compare the interview data.

Examples of the categories were implemented measures, barriers, future prospects, the company's energy vision, organisation with regard to energy issues, financing, resources, authority of the energy manager, information to employees, awareness, and cooperation.

Of the selected steel plants, seven had their own steel production and four only performed shaping processes. The number of employees at the plants ranged between 65 and 4600 , and the annual energy use ranged between $4 \mathrm{GWh}$ and $11 \mathrm{TWh}$ (see Table 3). The respondents had been employed at their companies between 4 and 40 years and the majority were engineers. Four of the 11 companies had joined the Swedish programme for improving energy efficiency in energy-intensive industry (PFE). These companies had implemented a standardised energy management system because that is one of the requirements for participating in the PFE programme. However, only one of the PFE participating companies had an energy manager working full-time with energy issues. Three of the companies in the study belonged to the same corporate group, two belonged to another corporate group, one was owned by two of the other companies in the study and the rest had no joint ownership with any of the other companies in the study.

Table 3. Characterisation of the studied steel plants.

\begin{tabular}{lcccccc}
\hline $\begin{array}{l}\text { Main production } \\
\text { process }\end{array}$ & $\begin{array}{l}\text { Number of } \\
\text { companies }\end{array}$ & $\begin{array}{l}\text { Production } \\
\text { (ktonnes/y) }\end{array}$ & Employees & $\begin{array}{l}\text { Annual } \\
\text { energy use } \\
\text { (GWh/y) }\end{array}$ & $\begin{array}{l}\text { Participate in } \\
\text { PFE (number } \\
\text { of companies) }\end{array}$ & $\begin{array}{l}\text { Full- } \\
\text { time } \\
\text { energy } \\
\text { manager }\end{array}$ \\
\hline $\begin{array}{l}\text { Iron ore-based } \\
\text { steel production } \\
\text { (BF-BOF or DRI) }\end{array}$ & 3 & $300-2100$ & $700-2500$ & $600-11100$ & 1 & 1 \\
$\begin{array}{l}\text { Scrap-based steel } \\
\text { production (EAF) }\end{array}$ & 4 & $100-400$ & $880-4600$ & $100-1000$ & 1 & 0 \\
$\begin{array}{l}\text { Rolling mill or wire } \\
\text { drawing }\end{array}$ & 4 & $34-2800$ & $65-2400$ & $4-2300$ & 2 & 2 \\
\hline
\end{tabular}

According to the definition of the European Commission (2003) a large enterprise has more than 250 employees and turnover of more than $€ 50$ million. One of the studied iron and steel companies could be characterised as a medium-sized enterprise, while the rest belonged to the group large enterprises.

In the interviews, the respondents gave subjective views on the subjects discussed, and other persons at the company might have a different opinion. At the same time, the positions of the interviewees and the fact that they influenced the company's work with energy efficiency measures made them interesting actors to interview. 


\section{Results and discussion}

Creating a continuous process of energy efficiency is a management issue as discussed above. The analysis below uses that as a starting point and will focus on energy efficiency from a process perspective.

\subsection{Barriers to improved energy efficiency}

When the respondents were asked to mention some implemented measures ${ }^{3}$ for improved energy efficiency at their company, their answers revealed a spectrum of measures (Table 4). All of the companies in the study recovered excess heat, and eight of them delivered heat to the municipal district heating system (DHS). However, it was stated in the interviews that there was potential to recover more excess heat. The respondents explained that barriers to increasing heat recovery were large investment costs, intermittent character of the heat flows, and seasonal variations in the DHS heat demand. Previous research has shown that barriers to low-grade heat recovery are a lack of infrastructure and financial support, high capital costs, and problems associated with a mismatch between the location of industrial processes and the potential users of the heat (Walsh and Thornley 2012). During the interviews, it was discovered that some of the existing heat cooperations with local DH distributers had been initiated due to contracts where the DH distributer invested in the infrastructure (pipes and heat exchangers) under the condition that they received all revenues from the sold heat during, for example, the first 10 years of operation. Heat distribution companies often have long-term energy strategies that allow for long payback periods, but the iron and steel industry often applies payback periods of less than 3 years. More heat cooperations of this type, where the heat distribution companies take the financial risk, might increase industrial excess heat delivery to the DHS. However, according to the respondents the excess heat streams left to be recovered are often the streams with the lowest recovery potential or the ones requiring complicated and/or expensive installations to recover. Therefore, it is recommended that heat recovery installations be planned and installed at the same time as new production equipment.

The respondents mentioned energy efficiency proposals that had been rejected, for example, intranet-based visualisation systems for energy use, new waste-heat boilers, cooperation with an external company to produce electricity from excess heat in an organic Rankine cycle, and employment of new staff that would work with energy issues. According to the respondents, the reasons for not implementing these measures were too long of a payback period ${ }^{4}$, lack of profitability, lack of staff to implement the measure, low priority given to energy management, low willingness to invest due to recession, risk of production disruption, lack of time, and fear of other people interfering in the management. These reasons were quite similar to those presented in other studies on barriers (Table 1), but the barrier "fear of other people interfering in the management" has not been seen in earlier research. This barrier was a result of territorial thinking in which those in charge of production opposed other people at the company having access to detailed energy monitoring of production processes. They did not want anyone interfering in their management of production. This attitude could result in less cooperation and knowledge sharing between different departments. It could also suggest that improved energy efficiency relies on the interests and

\footnotetext{
${ }^{3}$ The measures for improved energy efficiency reported are the ones the respondent knew of and thought worth mentioning.

${ }^{4}$ The investment criterion defined by the payback period is a risk indicator and not an economic feasibility indicator.
} 
priorities of the production managers, and this highlights the importance of a company having committed employees.

One respondent said that during the recession employees were not dismissed but instead used to optimise the company's energy system. A large part of the production was shut down and this offered an opportunity to improve the energy efficiency of idling. This is an example of costs being a driving force for taking action to improve energy efficiency.

Table 4. Implemented measures for improved energy efficiency mentioned by the respondents.

\begin{tabular}{|c|c|c|c|c|}
\hline Maintenance & $\begin{array}{l}\text { New } \\
\text { equipment }\end{array}$ & $\begin{array}{l}\text { Optimisation } \\
\text { of processes }\end{array}$ & $\begin{array}{l}\text { Energy } \\
\text { recovery }\end{array}$ & $\begin{array}{l}\text { New working } \\
\text { routines }\end{array}$ \\
\hline $\begin{array}{l}\text { Sealing } \\
\text { leakages in } \\
\text { compressed air } \\
\text { system }\end{array}$ & $\begin{array}{l}\text { Sub-metering } \\
\text { of energy use }\end{array}$ & $\begin{array}{l}\text { Optimisation } \\
\text { of idling }\end{array}$ & $\begin{array}{l}\text { Pre-heating of } \\
\text { scrap }\end{array}$ & $\begin{array}{l}\text { New routines } \\
\text { for operating } \\
\text { processes }\end{array}$ \\
\hline $\begin{array}{l}\text { Sealing of } \\
\text { furnace }\end{array}$ & $\begin{array}{l}\text { Energy- } \\
\text { efficient } \\
\text { burners in } \\
\text { furnaces }\end{array}$ & $\begin{array}{l}\text { Optimisation } \\
\text { of pump } \\
\text { system for } \\
\text { cooling water }\end{array}$ & $\begin{array}{l}\text { Heat-collector } \\
\text { over cooling } \\
\text { bed }\end{array}$ & $\begin{array}{l}\text { Knowledge- } \\
\text { sharing with } \\
\text { furnace } \\
\text { operators }\end{array}$ \\
\hline $\begin{array}{l}\text { Walls and } \\
\text { ceilings painted } \\
\text { with light } \\
\text { colours }\end{array}$ & $\begin{array}{l}\text { Frequency- } \\
\text { controlled } \\
\text { compressors }\end{array}$ & $\begin{array}{l}\text { Temperature } \\
\text { control of } \\
\text { furnace } \\
\text { operations }\end{array}$ & $\begin{array}{l}\text { Conversion } \\
\text { from steam to } \\
\text { hot water in } \\
\text { local heating } \\
\text { system }\end{array}$ & $\begin{array}{l}\text { Regular } \\
\text { inspection of } \\
\text { furnaces }\end{array}$ \\
\hline $\begin{array}{l}\text { Renovated } \\
\text { locker-rooms }\end{array}$ & $\begin{array}{l}\text { Frequency- } \\
\text { controlled } \\
\text { pumps }\end{array}$ & $\begin{array}{l}\text { Production- } \\
\text { controlled } \\
\text { hydraulic unit }\end{array}$ & $\begin{array}{l}\text { Recovery of } \\
\text { energy-rich } \\
\text { process gases }\end{array}$ & $\begin{array}{l}\text { Turning off } \\
\text { lights }\end{array}$ \\
\hline \multirow[t]{5}{*}{$\begin{array}{l}\text { Sealing of cold } \\
\text { storage }\end{array}$} & $\begin{array}{l}\text { Switching to } \\
\text { LED lights }\end{array}$ & $\begin{array}{l}\text { Temperature- } \\
\text { controlled } \\
\text { air-curtains }\end{array}$ & $\begin{array}{l}\text { Excess heat to } \\
\text { district } \\
\text { heating } \\
\text { network }\end{array}$ & $\begin{array}{l}\text { Program for } \\
\text { energy } \\
\text { conservation } \\
\text { in everyday } \\
\text { life }\end{array}$ \\
\hline & $\begin{array}{l}\text { Phase } \\
\text { compensation } \\
\text { in switchgear }\end{array}$ & $\begin{array}{l}\text { Optimisation } \\
\text { of control } \\
\text { systems }\end{array}$ & $\begin{array}{l}\text { Furnaces with } \\
\text { recuperators }\end{array}$ & $\begin{array}{l}\text { Considering } \\
\text { energy in } \\
\text { engineering } \\
\text { projects }\end{array}$ \\
\hline & Ceiling fans & $\begin{array}{l}\text { Larger filling } \\
\text { degree in } \\
\text { furnaces }\end{array}$ & $\begin{array}{l}\text { Recovery of } \\
\text { hot air from } \\
\text { filter }\end{array}$ & Energy audits \\
\hline & $\begin{array}{l}\text { Efficient } \\
\text { ventilation }\end{array}$ & & $\begin{array}{l}\text { Waste-heat } \\
\text { boiler }\end{array}$ & \\
\hline & $\begin{array}{l}\text { Fuel } \\
\text { conversion }\end{array}$ & & & \\
\hline
\end{tabular}


The majority of the energy efficiency measures implemented were associated with support processes, energy recovery, optimisation of operation and behavioural changes. Moreover, renovation of locker-rooms and painting walls and ceilings with light colours were primary done to improve working conditions of the employees and not to improve energy efficiency. None of the measures mentioned were related to the production processes of steelmaking in BF-BOF and EAF, and this was probably due to that these processes have minor potentials for improvements during the equipment's lifetime.

Two respondents stated that a well-prepared pre-study is a success factor for a positive decision with regard to investments in energy efficiency measures. However, they mentioned that lack of time and personnel sometimes prevented the initiation and execution of such pre-studies. In the author's opinion, it is unfortunate that it is not always prioritised to set aside time to conduct thorough prestudies because such a process might be worthwhile in the end. Six respondents said that the major limiting factor for taking action to improve energy efficiency was not money, but rather time and personnel resources and a lack of people with relevant education. They said that there was a need for more employees who could work with energy efficiency issues, and they requested personnel with higher education in the energy field. One energy manager said:

...there are many talented engineers, but they are not energy engineers /... / they can draw their diagrams, but somebody has to tell them how A plus B are linked. It is an educational question. It is very noticeable which educational pathway they have taken. When they have higher levels of education, they do not have these problems.

The need for personnel with higher education and a comprehensive view of the energy landscape has also been recognised in earlier research (see e.g. Thollander et al. 2007; Trianni and Cagno 2012). This finding indicates that the climate issue is not only becoming more important but also more complex.

Moreover, five respondents had experienced that suppliers of production equipment sometimes lacked competence with regard to a larger energy-system perspective, i.e. they had the required knowledge about engine classes but not of how to calculate and optimise process flows. The respondents said that large and established suppliers did not offer customer-adapted solutions, but instead it was up to the steel company to suggest energy-efficient solutions that considered the entire industrial energy system. Steel-making processes are complex and it would be advantageous if the supplier were familiar with the processes. However, one respondent stated that he/she had experienced that new actors who entered the market sometimes had a more innovative concept with flexible technical solutions that could be adapted to different customers. This finding can be related to the education issue, and it suggests that there is a need for adequate education across the production chain. These results were confirmed in earlier studies, e.g. Trianni and Cagno (2012) recognized that the industry experienced difficulty in finding the required external technical skills. However, in their study this was not considered as an important barrier. If the steel company lacks personnel with competence in energy systems issues, the difficulty in finding suppliers with this skill is even more problematic. In the worst-case scenario, the result is an energy inefficient installation that neither the steel company nor the supplier of the installation is aware of. This highlights the need for energy service companies that have competence in both industrial production processes and energy systems studies that could guide steel companies with no appointed energy manager. 
This study explores barriers experienced by energy managers and did not request the energy managers to rank predefined factors that they think could be barriers to energy efficiency. Therefore, the study complements previous research because many of the barriers that have been ranked as important in questionnaires in previous research were also reported as barriers that the energy managers in this study had experienced. The respondents highlighted lack of time as an important barrier to efficient energy management, and this barrier has also been found in previous research (Apeaning and Thollander 2013; Rohdin and Thollander 2006; Rohdin et al. 2007; Thollander et al. 2007; Thollander and Ottosson 2008; Trianni and Cagno 2012; Trianni et al. 2013a; Trianni et al. 2013c). The findings of this study would be representative for large energy-intensive industry branches.

\subsection{Responsibility and authority}

As mentioned, assigning climate change issues to an energy manager has been shown to influence companies toward more climate-friendly practices (Martin et al. 2012). Six of the companies had appointed a person to the position of energy coordinator. The other companies had not explicitly assigned the task of improved energy efficiency to one person but had embodied the task in the job assignments of several employees. The responsibility was either defined in a job description or had been self-imposed by employees because of their interests. The smallest steel company, which could be defined as a medium-sized company, was one of the companies with no appointed energy manager. Three of the respondents worked full-time with energy management; four worked $50 \%$ of their time; and the others spent $5 \%-10 \%$ of their working time on energy-related issues. It is noteworthy, that the energy managers working full-time with energy management did not experience lack of time and personnel. Two respondents were on their company's board of directors, which is usually beneficial for energy efficiency to be anchored in a company's processes and routines. However, only one company had an appointed energy manager with clear delegation and responsibility, who was fully integrated into management structure.

Six of the studied companies had an energy committee where management personnel met up to four times a year and discussed proposals for improved energy efficiency and made decisions about the company's energy strategy. The energy committees ensured the continuity of an organised and structured programme of energy-saving projects. Such internal networks can also contribute to the spread of an energy-efficient culture in the company and engage more people in the issues. Networking within the company has been found in previous research to be a driving force for improved energy efficiency (Apeaning and Thollander 2013; Thollander et al. 2013; Thollander and Ottosson 2008). An energy committee could also contribute to overcoming the territorial barrier analysed in Section 5.1. This should be a prestigeless meeting forum where managers help and inspire each other with open minds and mutual respect.

One of the respondents told that energy issues related to production and support processes were administrated separately. This might reduce the barrier of production managers not prioritising energy issues, but it might also be a barrier to an efficient industrial energy system if energy use in support processes and production processes are optimised separately. This was highlighted by the respondent who said that different departments must have a dialogue and cooperate in order to find the most optimal energy system. However, in that person's experience this dialogue was often insufficient or non-existent. In an energy management perspective, cooperation and dialogue is vital to achieving continuous improvements. Therefore, again, an energy committee can be an important 
meeting place where the energy manager and representatives from all departments can discuss energy-saving measures.

When the respondents were asked to specify their job assignments with regard to energy issues, the most frequent answer was that they were to coordinate the energy work at the company. They also mentioned contacting authorities, reporting on legislative compliance, collecting and compiling energy-use statistics, developing and following up on energy goals, performing energy audits, advising on investment decisions, working with the energy-management system, and analysing economic calculations and technical specifications. These tasks are all important components of an effective energy management system as described in Section 2.1. All the companies monitored trends in energy use and ten had performed energy audits. One respondent also expressed that environmental legislations was a driving force for collecting energy use statistics and analyse and follow up the company's energy performance.

To engage in efficient energy management, it is important that the energy manager has support from senior management, see, e.g. (Caffall 1995; Mashburn 2005). The results of this study indicate that the majority of the respondents had limited power to make decisions about energy-efficiency projects. Three respondents had the authority to make such decisions. The others had to present proposals for the head of the department where the measure would be implemented or to the senior management. In general, they experienced that senior management listened to their proposals and opinions. Five companies reported an energy policy with a long-term energy strategy of more than three years. For a successful energy management system, the energy manager should have the authority to be part of business planning, and 10 of the respondents said that they could influence long-term strategic decisions. Three of the respondents expressed a wish to be able to influence more.

During the interviews, it was found that energy managers who had been employed at the company many years and had worked with other job assignments saw this as an advantage in their position as energy manager. As one energy manager said:

I have worked down there in production and then when I came up here and had some broader perspective, it was actually quite fun. You see things from different perspectives depending on where you work. This is important to have in mind when you implement your measures, because then you know that the ones working down in production, they struggle every day. Things break and then I come, some wise guy, and start to poke with energy. You must do it in a proper way.

Moreover, having knowledge about the production processes gives the energy manager authority in the eyes of the production employees. During the interviews, it was mentioned that it was easier to convince employees to accept proposals for energy-saving actions if the proposals were put forward by people who were considered to know what they were talking about. This can be related to the importance of organizing an energy team that can support the energy manager with supplementary skills and knowledge.

\subsection{Prioritising of energy efficiency practices}

In general, energy investments at the companies studied were prioritised as number three or four in budget planning. One company also had an energy strategy that stated that energy was a core business. Recognising that energy is important has previously been identified as a factor contributing 
to successful energy management (Caffall 1995). Nine respondents thought that their company prioritised energy issues, even if some also felt that energy should be more highly prioritised. According to the respondents, energy costs were one of the driving forces for prioritising energy issues. Cost reduction from lowered energy use has previously been identified as an important driving force for improved energy efficiency in industry (Apeaning and Thollander 2013; Brunke et al. 2014; Christoffersen et al. 2006; Thollander and Ottosson 2008; Thollander et al. 2013). The respondents who thought that their company did not prioritise energy issues supported this by noting the fact that there had been reductions in the numbers of employees involved with energy efficiency issues. It is noteworthy that these personnel reductions occurred during a period of recession. In addition, these respondents experienced that the board of directors did not listen to them. They were not directly subordinate to the CEO and felt that their proposals and information did not reach the CEO. Consequently, the companies sometimes did not make decisions with regard to investment in measures for improved energy efficiency. This is supported by earlier research. For example, Mashburn ( 2005) stated that a hierarchal proximity between the energy manager and the CEO resulted in more climate-friendly practices.

Decisions about implementation of energy efficiency measures were often made by the facility manager in charge of the production processes in which the measure would be implemented, and they did not always make the measures a priority. One energy manager said:

Sometimes this is not so good. In particular, improvements in support processes usually end up way down on the production manager's list.

The risk of disrupting production has been shown in previous research to be an important barrier for improved energy efficiency in energy-intensive industry (Brunke et al. 2014; Lee 2014; Rohdin et al. 2007; Thollander and Ottosson 2008). However, this should not be a barrier for energy efficiency measures in support processes and it could be argued that it should be easier to get a decision to implement energy-saving measures in support processes than in production processes. However, the respondents said that the production managers' primary priorities were usually on optimising production processes with regard to quantity and quality of the product and not on improving energy efficiency. A separation of the administration of support and production processes might increase the prioritisation of the energy efficiency of support processes, but as noted in Section 5.2 this arrangement demands cooperation between departments to achieve efficiency in total energy use.

At the time of the interviews, none of the companies had a budget designated for energy investments. However, six years earlier one company reserved $5 \%$ of its investment budget for energy efficiency investments but stopped doing so after a re-organisation and replacement of personnel. The majority of those interviewed did not ask for a designated budget. On the contrary, one respondent thought that it would be more advantageous to allow a longer payback period for energy investment as compared to other investments than to reserve a budget for energy efficiency investments. This strategy had been implemented at some of the companies. This suggests that the type of strategy chosen for promoting energy efficiency investments might not be crucial for successful energy management but that having the strategy anchored in the company is. 


\subsection{Engaging employees}

Ates and Durakbasa (2012) pointed out the importance of education, training, and capacity building for effective energy management and improved energy efficiency in industry. All the companies in this study offered some kind of education for new employees in which environmental and energy issues were included as a minor part. Additionally, six of the companies had more specific education about energy and energy efficiency for employees. In general, this education was directed to persons in specific positions such as managers, project leaders, and engineers. One respondent said that their company planned to repeat and update the education every five or seven years, but the other respondents did not report such scheduled continuity. It would be valuable for companies to give personnel on-going training and education to update their skills and to keep awareness of energy issues alive.

Earlier research has stated that successful energy management includes engaging all employees in improving energy efficiency (Christoffersen et al. 2006; Jørgensen et al. 2008; Mashburn 2005), but this was not a common practice in the companies in this study. The majority of the respondents thought that their companies could increase their efforts to engage employees. The respondents said that they were usually the ones who suggested measures for improved energy efficiency, but occasionally employees from, for example, the production and media departments could make additional proposals. The companies had improvement proposal schemes, but none of the schemes were specified for energy efficiency proposals exclusively, and in general employees were not explicitly invited to send in proposals. Even though three companies offered the employee a share of the profit from implementing the proposed energy saving measures, the schemes were not extensively utilised. This implies that the companies studied had not succeeded in involving all employees in energy management. One respondent said that he/she had never received proposals from employees on the floor. The person speculated on whether this was due to the fact that he/she had no time to visit the production area. Again, lack of time often proves to be a barrier for improved energy efficiency. In contrast, two respondents who spent a lot of time with the people working in production said that they had a dialogue where information was exchanged and where they could receive tips about energy-saving projects. Mashburn (2005) also recommended that energy managers should spend time talking to the employees.

Two respondents thought that it was not important to engage all employees in energy management. They thought that it was more important to find improvements in production processes than to change the behaviour of employees. In contrast, another respondent reported that his/her company had had people walk around in the production area asking employees about energy use and that afterwards they had recorded a decrease in energy use. The respondent concluded that simply by making people aware of the issue their behaviour became more energy efficient. This is confirmed by previous research that showed that a barrier to improved energy efficiency is a lack of staff awareness (Lee 2014; Rohdin and Thollander 2006; Thollander et al. 2007; Thollander and Ottosson 2008; Trianni and Cagno 2012; Trianni et al. 2013a). Three of the companies were part of the same corporate group that had started a project to involve all employees in a scheme for continuous improvement and implementation of best practices. Practices for improved energy efficiency were included in the scheme and one of the companies in the corporate group had started a pilot project on energy savings in everyday life. The purpose was to increase awareness among all employees and to introduce energy-efficient behaviour, such as turning off lights and operating furnaces efficiently. 
By introducing a perspective in which every energy-saving activity is regarded as important, the company builds an energy-efficient culture. It has been shown that when senior management does not promote energy-saving measures the behaviour of the employees is negatively affected (Nisiforou et al. 2012).

Two companies had introduced a system in which employees working in production departments were encouraged to register as energy hosts, and their function was to report to the energy coordinator about how the operations in their department were performed with regard to energy use. The energy hosts also received information from the energy coordinator and attended official meetings scheduled about twice a year where they and the energy coordinator went through what had happened since the last meeting. This system functioned well and one respondent explained its success by saying that involving employees introduced a collective responsibility for improved energy efficiency. This can be correlated to the fact that effective energy management includes engaging and motivating employees. One respondent said that it is easier to have a dialogue and engage employees if they are in small groups than if they are in large groups:

... and my strategy now is to go out and talk, as I do with you here, in the group. Therefore, I am out in the departments visiting the staff coffee rooms because I get much better feedback when it is up to five persons. Because when I have 50 persons then $99 \%$ keep quiet and 1\% talk. Now everybody opens their mouth. /.../It takes time, but I get to know a lot of faces. And then I tell them to come back to me when they have some ideas about what is bad and what is good.

This strategy allows everybody to be visible and acknowledged and, therefore, to be more motivated to engage in energy savings. This way of informing personnel might be more time-consuming, but the respondent said that the benefits of this approach outweighed the effort involved. One barrier to companies adopting this type of comprehensive information exchange might be that many energy managers do not work full-time with energy issues and have an overall high workload.

It has previously been stated that having people with real ambition is an important driving force for implementing energy-saving measures (Brunke et al. 2014; Rohdin and Thollander 2006; Rohdin et al. 2007; Thollander et al. 2007; Thollander and Ottosson 2008). The importance of dedicated and interested employees was also expressed during the interviews. For example, one respondent had worked on improving energy efficiency out of personal interest and the company had implemented several energy saving projects because of this engagement. Another respondent described a colleague with commitment and authority who made things happen:

It is based on two things; the first is that he is very experienced and interested. This is what he thinks is fun, and he has worked all over the place so he knows how it works. And the second is that he has been head of the plant. So when he comes and says "Let us do it like this", everybody nods and does as he says. They do not question whether his ideas or suggestions are feasible and sound.

This example shows the importance of having a champion in the company who makes things happen. The role of the champion can be correlated to the advantage of having knowledge about, and experience in, the production processes. Conversely, a person without a commitment to energy improvements can become a barrier to implementation. The interviews revealed that this type of 
person exists at every level of the company hierarchy. One respondent had worked with such a person:

Other people /.../ they read this little routine in the management system like the devil reads the Bible and they acknowledge that, "Oh well I have to do an LCC analysis for a couple of different options, but I can decide what alternatives I will choose. I know what I want to do so I invent an alternative that I know is worse and so I compare with that. Then I hand in the results and I have fulfilled the routines."

The fact that persons without commitment can be barriers to successful energy management has been recognised in previous research (see, for example, (Trianni et al. 2013a)). They might be as much a barrier to energy savings as committed persons are a driving force, and this highlights the importance of engaging all employees in the process of improving energy efficiency.

\subsection{Networking}

Networking within the company or group, in the same industrial sector, and between industry and suppliers has previously been shown to be important for effective energy management (Apeaning and Thollander 2013; Thollander and Ottosson 2008; Trianni et al. 2013a; Christoffersen et al. 2006). Parker (2013) showed that human interactions, rather than written policies and procedures, affected the way employees interpreted their tasks and roles. Okazaki and Yamaguchi (2011) recommended actions to accelerate the transfer and dissemination of energy efficiency technologies in the steel industry. They stated that the key factors for removing barriers are information sharing and utilisation of expert's knowledge and experiences. An important meeting place and arena for networking for the Swedish steel industry's energy managers is the Energy Council at Jernkontoret. The respondents were members of the Council and all except one regarded that membership as valuable for their profession. Above all they appreciated the benchmarking and news updates that Jernkontoret provides. It was considered valuable to receive information about new legislations, EUpolicies, benchmarking, and political trends in a condensed format. Moreover, they regarded the Energy Council a valuable network where members could exchange ideas and discuss common strategies with regard to topics such as the EU Emission Trading Scheme (EU-ETS) and future legislation. However, in general, the energy managers at smaller steel companies did not find as much value in being a member as did energy managers at larger ones. Representatives from the smaller companies said that the Council mainly discussed global issues, which were of minor interest to them. One respondent even thought that Jernkontoret did not listen to the smaller steel companies but only to the larger ones.

All of the respondents, with one exception, were members of the ENET-Steel network. However, in some cases the respondents were not sure if they were a member until the network was described in more detail. The respondents who had attended network meetings thought they functioned well and provided an opportunity to exchange knowledge and get ideas and inspiration. They particularly appreciated meetings with site visits where "good examples" were presented. Other studies of energy efficiency networks have been published, and they show similar results. For example, Jochem and Gruber (2007) evaluated an energy efficiency network with companies from German industrial and service sectors. They found that most of the companies considered the meetings helpful to expand their horizons and discuss new topics and that the exchange of experiences inspired actions for improved energy efficiency. The members thought that it was important to have open discussions 
where everybody could share both successes and failures. Amundsen (2000) analysed the Norwegian Industrial Energy Efficiency Network, which is a network of 540 companies. The network provided its members with motivation, knowledge, and obligation. According to Amundsen (2000), 80\% of the companies experienced that participation in the network had contributed in a positive way to the leaders' and employees' engagement in improved energy efficiency. He concluded that networking among companies is an effective way of achieving energy savings in the industry.

During the interviews, several respondents mentioned other energy managers at Swedish steel plants by name and declared that they were impressed by their work with improved energy efficiency. Their goal was to accomplish the same results. This confirms the importance of networking as a means of spreading ideas and experiences about energy-saving measures. However, in some cases long travel distances prevented the respondents from attending meetings. Another barrier was lack of time because of a high workload. Moreover, two respondents said inspiration often faded after they returned home and the ideas ended up in a drawer waiting for them to have the time to work with them. As can be seen, lack of time is not only a barrier to improved energy efficiency at the company but also a barrier to the exchange of knowledge with other companies. Only one respondent used the chat site on the Internet, and even then only occasionally. The others said they did not use it because they did not chat on websites, the issues discussed were too technical, the design of the site was not user-friendly, and it was easier and faster to search for answers on Google or to call somebody.

As the results show, the network meetings contributed to valuable knowledge transfer and members could be inspired by each other or by invited speakers. They also provided a welcome opportunity to discuss problems with colleagues facing the same challenges. However, one respondent declared that at some point it would become harder to come up with new meeting agendas that would attract a majority of the network members. This suggests that in deciding on the frequency of networking meetings there has to be a compromise between the need to space out meetings so new and interesting issues can be presented and the importance of ensuring continuity to keep the network active.

None of the respondents felt that secrecy hindered them from discussing opportunities and problems with regard to improved energy efficiency. They all thought the open atmosphere at the network meetings resulted from the fact that Swedish steel companies have their own niches of steel products and do not compete and that energy issues are a common concern that do not involve secret production processes. The companies were not reluctant to share knowledge on how to reduce energy costs. Instead, there are several joint research projects administered by Jernkontoret, with the aim to reduce energy use in the iron and steel industry, where different steel companies work together. However, some suppliers were also members of the network and sometimes the respondents saw this as a barrier to an open discussion. The respondents did say, however, that it was interesting to have suppliers present new equipment at network meetings. According to the person responsible for ENET-Steel, the ambition is to extend the network to include actors from other industrial sectors in Sweden. This might be a good idea because Palm and Thollander (2010) recommended communication between social networks in different sectors to stimulate new social arrangements in which actors can share good examples with regard to improved energy efficiency. 
The companies also exchanged information and cooperated through other networks. Several of the companies belonged to corporate groups with facilities in other countries with which they cooperated. However, one respondent said that their company had had no international relationships with regard to energy until they had a new owner who was very ambitious about energy issues. This had inspired the personnel at the company. The owner also strived to increase cooperation among companies in the organisation to achieve energy savings. Representatives from different companies within the division shared experiences, and this example confirms the importance of senior management support for efficient energy management. According to Ates and Durakbasa (2012), relations with neighbours, customers, and environmental organisations can pressure a company to take actions toward adopting more environment- and climate-friendly practices. To have relationships with industry in foreign countries or to have a foreign owner also affects energy management practices. Ates and Durakbasa (2012) stated that Turkish companies owned by investors from developed countries had relatively advanced energy management practices.

The interviews revealed that the respondents also created their own networks with actors having common interests (e.g. other industries and energy companies in the region) in which electricity purchasing and heat recovery were discussed. One respondent pointed out the importance of cooperation:

... and we have many common interests with regard to heat recovery. How should a future strategy be formulated? Because, every decision in this system affects all three companies ...

This statement suggests that networking is important for improved energy efficiency from a larger system perspective and can result in an efficient regional energy system. However, industrial energy efficiency and heat cooperation between industry and DH distributers can counteract each other. Improved energy efficiency can result in less industrial excess heat available for delivery to the DHS, and built-in systems for industrial excess heat delivery can hinder investment in other energy efficiency measures.

\subsection{Summary of barriers and driving forces}

The barriers to and driving forces for improved energy efficiency shown in this study were selfexperienced by the energy managers interviewed and there may be barriers and driving forces, which were not expressed during the interviews. The interviewees have not ranked the relative importance of the barriers and drivers since different situations were affected by different barriers or drivers. Table 5 and Table 6 summarise and categorise the barriers and drivers experienced and mentioned by the respondents. 
Table 5. Barriers experienced by the energy managers interviewed. The barrier may have its origin within the firm (I) or outside the firm (E).

\begin{tabular}{|c|c|}
\hline Category & Barrier \\
\hline \multirow[t]{2}{*}{ Technology-related barrier } & $\begin{array}{l}\text { Seasonal variations in the DHS heat demand } \\
\text { (barrier to industrial excess heat recovery and } \\
\text { export to DHS) (E) }\end{array}$ \\
\hline & $\begin{array}{l}\text { Intermittent excess heat flow (barrier to } \\
\text { industrial excess heat recovery and export to } \\
\text { DHS) (I) }\end{array}$ \\
\hline \multirow[t]{4}{*}{ Organisational } & Lack of time (I) \\
\hline & Lack of personnel (I) \\
\hline & $\begin{array}{l}\text { Lack of direct contact between energy manager } \\
\text { and senior management/CEO (I) }\end{array}$ \\
\hline & Lack of internal dialogue (I) \\
\hline \multirow[t]{2}{*}{ Information barriers } & $\begin{array}{l}\text { Information not clear by the technology suppliers } \\
\text { (E) }\end{array}$ \\
\hline & Lack of pre-studies (I) \\
\hline \multirow[t]{2}{*}{ Economic } & Risk of production disruption (I) \\
\hline & $\begin{array}{l}\text { Investment criterion - Too long payback period } \\
\text { (I/E) }\end{array}$ \\
\hline Market & Low willingness to invest due to recession $(\mathrm{E})$ \\
\hline \multirow[t]{5}{*}{ Behavioural } & Production managers have other priorities (I) \\
\hline & Other priorities for capital investments (I) \\
\hline & $\begin{array}{l}\text { Personnel without commitment for energy } \\
\text { efficiency (I) }\end{array}$ \\
\hline & Personnel resist change (I) \\
\hline & Territorial thinking (I) \\
\hline \multirow[t]{2}{*}{ Barriers related to competence } & $\begin{array}{l}\text { Technology suppliers lack energy competence } \\
\text { and holistic view (E) }\end{array}$ \\
\hline & $\begin{array}{l}\text { Lack of people with higher education in the } \\
\text { energy field (I/E) }\end{array}$ \\
\hline Awareness & $\begin{array}{l}\text { Lack of awareness of the potential of engaging } \\
\text { employees (I) }\end{array}$ \\
\hline
\end{tabular}


Table 6. Driving forces for improved energy efficiency experienced by the energy managers interviewed. The driving force may have its origin within the firm (I) or outside the firm (E).

\begin{tabular}{ll}
\hline Category & Driving force \\
\hline $\begin{array}{l}\text { Organisational } \\
\text { Information drivers }\end{array}$ & $\begin{array}{l}\text { Cooperation within the corporate group (I/E) } \\
\text { Networking with other energy managers: } \\
\text { knowledge and information sharing (E) } \\
\text { Well-prepared pre-studies (I) } \\
\text { Economic }\end{array}$ \\
$\begin{array}{l}\text { Cost reduction from lowered energy use (I) } \\
\text { Pehavioural }\end{array}$ & $\begin{array}{l}\text { Senior management prioritises energy issues (I) } \\
\text { Energy manager has knowledge and experience } \\
\text { of production processes (I) }\end{array}$ \\
Awareness & Making employees aware of energy issues (I) \\
Government/politics & Compliance with regulatory issues (E) \\
\hline
\end{tabular}

Three of the four top-ranked barriers and three of the four top-ranked drivers for improved energy efficiency in Swedish iron and steel industry found by Brunke et al. (2014) were also mentioned by the respondent in this study. The exceptions were the barrier "lack of access to capital" and the driving force "long-term energy strategy". The respondents did not experience a lack of access to capital, but rather that other investments were prioritised before energy investments. Moreover, long-term energy strategy was not mentioned as a driving force. However, five companies had a long-term energy strategy of more than three years. The majority of the barriers and drivers mentioned by the respondents have their origin within the company and could therefore be related to the company's energy management practices. The barriers with their origin outside the company were, with two exceptions, related to imperfections of the technology suppliers. This could be correlated to the complex processes of iron and steelmaking; the technology suppliers often showed a lack of knowledge about steel production processes. The external driving forces "cooperation within the corporate group" and "networking with other energy managers" rely on that people are willing to contribute with their knowledge and experience and have an open mind to new ideas. Some recommendations of actions to improve energy efficiency are presented below in "Conclusions and recommendations".

\section{Conclusions and recommendations}

The majority of the barriers to improved energy efficiency experienced by the respondent had their origin within the company, which implies that in theory they may be removed by sound energy management practices. Based on the results of the interview study some conclusions and recommendations for a successful energy management are given. 
In general, the respondents considered lack of time to be a bigger barrier to improved energy efficiency than lack of access to capital. Only three out of the eleven companies had assigned a person to work full-time with energy management, and some of the energy managers were frustrated with not having enough time to work with energy issues. Only a minority of the energy managers had the authority to make decisions about energy-saving projects. One rather obvious recommendation to these companies would be to employ a full-time energy manager to accomplish effective energy management and develop optimised energy systems. It could also be advised to employ more people to work with energy issues at the company, under the leadership of the energy manager. The energy manager should, also organise an energy committee with decision-making power in which representatives from different departments could discuss potential projects and energy strategies. It is also important that the company prioritise initiation and completion of prestudies of potential energy-saving projects because a well performed pre-study lays the foundation for a positive investment decision.

The interviews revealed that the companies lacked personnel with education in energy engineering. By employing energy engineers and assigning them positions in different departments in the organisation, the energy issue can be integrated into purchasing, production management, and project planning. Employees with different competences, perspectives, and ideas working together could result in a combination of efficient production management and efficient energy management.

The interviews showed that people without commitment to energy savings could be a barrier to improved energy efficiency and even oppose the efforts to introduce efficient energy management. It is hard to change people's attitudes, but perhaps education and motivation campaigns that introduce an energy-efficient culture in the company could reduce the negative attitude toward energy savings. Positive peer pressure could at best affect these people to engage in energy-saving actions.

Most of the companies in this study prioritised energy investments as number three or four in their budget planning. In order to increase the number of investments in energy-efficiency measures, it is advised that the companies allow a payback period longer than three years for energy investments or designate a budget for energy-saving measures that can be administered, for example, by an energy committee. Moreover, the payback period is a risk measure and not a profitability measure. It is therefore recommended that the companies, in addition to payback period, use internal rate of return and/or net present value as financial criterion for investments in energy efficiency measures.

Employees are an untapped resource in energy management, and the majority of the respondents believed that their companies could do better at engaging employees in improving energy efficiency. The author suggests that the companies introduce education programmes for employees in energy issues and with scheduled continuity. In order to visualise energy use and energy saving measures, up-to-date energy use statistics, key energy performance indicators, and good examples of implemented energy efficiency measures should be presented on company intra-nets and on screens strategically placed in areas such as the cafeteria and in conference and meeting rooms. The board of directors and the energy committee should be role models for energy-saving behaviour, and every energy-saving action should be encouraged even if they seem small in comparison to the steel plant's total energy use. In this way, energy-efficient behaviour would become common practice among the employees. Employees should be encouraged to contribute with ideas and suggestions for energy 
efficiency measures, and the concept of energy hosts, as practiced at two of the studied steel companies, should be implemented at other steel plants because this has proven to function well.

The respondents regarded networking as valuable for sharing knowledge, exchanging information, and finding ideas and inspiration for energy-saving projects. However, lack of time often made inspiration fade and prevented the respondents from implementing the ideas. In order to develop productive networking, the energy issue must be more prioritised at the companies in terms of having more personnel assigned to work with energy saving projects.

In conclusion, Swedish steel companies regard improved energy efficiency as important but have much work left to do in this area. For example, vast amounts of excess heat are not recovered and more efforts could be put into engaging employees in order to introduce a culture of energy efficiency. Lack of time seems to be a major barrier for efficient energy management as well as for productive networking.

\section{Acknowledgements}

The work has been carried out under the auspices of the Energy Systems Programme, which is financed by the Swedish Energy Agency. The work was co-financed by Göranssonska Fonden, which is a trust fund. The author wishes to thank the interviewees for their participation and Jenny Palm and Mats Söderström at Linköping University for their valuable comments on this paper.

\section{References}

Abdelaziz, E. A., Saidur, R., \& Mekhilef, S. (2011). A review on energy saving strategies in industrial sector. Renewable and Sustainable Energy Reviews, 15(1), 150-168.

Amundsen, A. (2000). Networking among companies represents a potential for $\mathrm{CO} 2$ reduction. Journal of Cleaner Production, 8(6), 495-501.

Apeaning, R. W., \& Thollander, P. (2013). Barriers to and driving forces for industrial energy efficiency improvements in African industries - a case study of Ghana's largest industrial area. Journal of Cleaner Production, 53, 204-213.

Ates, S. A., \& Durakbasa, N. M. (2012). Evaluation of corporate energy management practices of energy intensive industries in Turkey. Energy, 45(1), 81-91.

Backlund, S., Thollander, P., Palm, J., \& Ottosson, M. (2012). Extending the energy efficiency gap. Energy Policy, 51, 392-396.

Bloom, N., Genakos, C., Martin, R., \& Sadun, R. (2010). Modern Management: Good for the Environment or Just Hot Air? Economic Journal, 120(544), 551-572.

Brunke, J.-C., Johansson, M., \& Thollander, P. (2014). Empirical investigation of barriers and drivers to the adoption of energy conservation measures, energy management practices and energy services in the Swedish iron and steel industry. Journal of Cleaner Production, doi:http://dx.doi.org/10.1016/j.jclepro.2014.04.078.

Bunse, K., Vodicka, M., Schönsleben, P., Brülhart, M., \& Ernst, F. O. (2011). Integrating energy efficiency performance in production management - Gap analysis between industrial needs and scientific literature. Journal of Cleaner Production, 19(6-7), 667-679.

Caffall, C. (1995). Learning form experience with Energy Management in Industry. CADDET Analyses Series No. 17: CADDET Sittard, the Netherlands. 
Cagno, E., \& Trianni, A. (2013). Exploring drivers for energy efficiency within small- and medium-sized enterprises: First evidences from Italian manufacturing enterprises. Applied Energy, 104, 276285.

Cagno, E., Worrell, E., Trianni, A., \& Pugliese, G. (2013). A novel approach for barriers to industrial energy efficiency. Renewable and Sustainable Energy Reviews, 19, 290-308.

Christoffersen, L. B., Larsen, A., \& Togeby, M. (2006). Empirical analysis of energy management in Danish industry. Journal of Cleaner Production, 14(5), 516-526.

De Groot, H. L. F., Verhoef, E. T., \& Nijkamp, P. (2001). Energy saving by firms: Decision-making, barriers and policies. Energy Economics, 23(6), 717-740.

Deming, W. E. (2000). Out of the crisis: The MIT Press, Massachusetts.

European Commission (2003). Commission recommendation of 6 May 2003 concerning the definition of micro, small and medium-sized enterprises. Official Journal of the European Union.

European Commission (2010). Final Report of the SET-Plan workshop on Technology Innovations for Energy Efficiency and Greenhouse Gas (GHG) emissions reduction in the Iron and Steel Industries in the EU27 up to 2030.

European Commission Eurostat (2013). Europe 2020 indicators.

epp.eurostat.ec.europa.eu/portal/page/portal/europe_2020_indicators/headline_indicators. Accessed 29 May 2013.

Fleiter, T., Worrell, E., \& Eichhammer, W. (2011). Barriers to energy efficiency in industrial bottom-up energy demand models-A review. Renewable and Sustainable Energy Reviews, 15(6), 30993111.

Hasanbeigi, A., Menke, C., \& du Pont, P. (2010) Barriers to energy efficiency improvement and decision-making behavior in Thai industry. Energy Efficiency, 3(1), 33-52.

International Organization for Standardization (2011). Win the energy challenge with ISO 50001. www.iso.org/iso/iso_50001_energy.pdf. Accessed 12 June 2013.

Jaffe, A. B., \& Stavins, R. N. (1994). The energy-efficiency gap What does it mean? Energy Policy, 22(10), 804-810.

Jernkontoret (2014). Jernkontoret - the Swedish Steel Producers' Association. www.jernkontoret.se/english/index.php. Accessed 12 February 2014.

Jochem, E., \& Gruber, E. (2007). Local learning-networks on energy efficiency in industry - Successful initiative in Germany. Applied Energy, 84(7-8), 806-816.

Jørgensen, F., Hyland, P., \& Busk Kofoed, L. (2008). Examining the role of human resource management in continuous improvement. International Journal of Technology Management, 42(1/2), 127-142.

Kvale, S. (1996). Interviews: An introduction to qualitative research interviewing. California: Sage Publications Inc.

Lee, K. H. (2014). Drivers and barriers to energy efficiency management for sustainable development. Sustainable Development. DOI: 10.1002/sd.1567.

Martin, R., Muûls, M., de Preux, L. B., \& Wagner, U. J. (2012). Anatomy of a paradox: Management practices, organizational structure and energy efficiency. Journal of Environmental Economics and Management, 63(2), 208-223.

Mashburn, W. H. ( 2005). Effective energy management. In W. C. Turner (Ed.), Energy management handbook fifth ed. (pp. 9-22). Georgia: The Fairmont Press Inc.

Miles, J. A. (2012). Management and Organization Theory : A Jossey-Bass Reader. Hoboken, NJ, USA Wiley 
Nisiforou, O. A., Poullis, S., \& Charalambides, A. G. (2012). Behaviour, attitudes and opinion of large enterprise employees with regard to their energy usage habits and adoption of energy saving measures. Energy and Buildings, 55, 299-311.

Okazaki, T., \& Yamaguchi, M. (2011). Accelerating the transfer and diffusion of energy saving technologies steel sector experience-Lessons learned. Energy Policy, 39(3), 1296-1304.

Palm, J., \& Thollander, P. (2010). An interdisciplinary perspective on industrial energy efficiency. Applied Energy, 87(10), 3255-3261.

Parker, T. (2013) The view from below - a management system case study from a meaning-based view of organization. Journal of Cleaner Production, 53, 81-90.

Pye, M., \& McKane, A. (2000). Making a stronger case for industrial energy efficiency by quantifying non-energy benefits. Resources, Conservation and Recycling, 28(3-4), 171-183.

Rohdin, P., \& Thollander, P. (2006). Barriers to and driving forces for energy efficiency in the nonenergy intensive manufacturing industry in Sweden. Energy, 31(12), 1500-1508.

Rohdin, P., Thollander, P., \& Solding, P. (2007). Barriers to and drivers for energy efficiency in the Swedish foundry industry. Energy Policy, 35(1), 672-677.

Sardianou, E. (2008). Barriers to industrial energy efficiency investments in Greece. Journal of Cleaner Production, 16(13), 1416-1423.

Sivill, L., Manninen, J., Hippinen, I., \& Ahtila, P. (2013). Success factors of energy management in energy-intensive industries: Development priority of energy performance measurement. International Journal of Energy Research, 37, 936-951.

Sorrell, S., Schleich, J., Scott, S., O’Malley, E., Trace, F., Boede, E., Ostertag, K., Radgen, P. (2000). Reducing barriers to energy efficiency in public and private organizations. www.sussex.ac.uk/Units/spru/publications/reports/barriers/final.html. Accessed 12 June 2013.

Sudhakara Reddy, B. (2013). Barriers and drivers to energy efficiency - A new taxonomical approach. Energy Conversion and Management, 74, 403-416.

Swedish Energy Agency (2012). Energy in Sweden - facts and figures 2012 www.energimyndigheten.se/en/Facts-and-figures1/Publications/. Accessed 12 June 2013.

Swedish Energy Agency (2013a). Energiläget 2013 [Energy in Sweden 2013].

Swedish Energy Agency (2013b). Targets with regard to energy use in Sweden and the EU [Mål rörande energianvändning i Sverige och EU] energimyndigheten.se/sv/Offentligsektor/Tillsynsvagledning/Mal-rorande-energianvandning-i-Sverige-och-EU/ [in Swedish]. Accessed 29 May 2013.

Thollander, P., Backlund, S., Trianni, A., \& Cagno, E. (2013). Beyond barriers - A case study on driving forces for improved energy efficiency in the foundry industries in Finland, France, Germany, Italy, Poland, Spain, and Sweden. Applied Energy, 111, 636-643.

Thollander, P., Danestig, M., \& Rohdin, P. (2007). Energy policies for increased industrial energy efficiency: Evaluation of a local energy programme for manufacturing SMEs. Energy Policy, 35(11), 5774-5783.

Thollander, P., \& Ottosson, M. (2008). An energy efficient Swedish pulp and paper industry Exploring barriers to and driving forces for cost-effective energy efficiency investments. Energy Efficiency, 1(1), 21-34.

Thollander, P., \& Ottosson, M. (2010). Energy management practices in Swedish energy-intensive industries. Journal of Cleaner Production, 18(12), 1125-1133. 
Trianni, A., \& Cagno, E. (2012). Dealing with barriers to energy efficiency and SMEs: Some empirical evidences. Energy, 37(1), 494-504.

Trianni, A., Cagno, E., Thollander, P., \& Backlund, S. (2013a). Barriers to industrial energy efficiency in foundries: a European comparison. Journal of Cleaner Production, 40, 161-176.

Trianni, A., Cagno, E., \& Worrell, E. (2013b). Innovation and adoption of energy efficient technologies: An exploratory analysis of Italian primary metal manufacturing SMEs. Energy Policy, 61, 430-440.

Trianni, A., Cagno, E., Worrell, E., \& Pugliese, G. (2013c). Empirical investigation of energy efficiency barriers in Italian manufacturing SMEs. Energy, 49, 444-458.

US Department of Energy - Advanced Manufacturing Office (2013). DOE eGuide for ISO 50001 ecenter.ee.doe.gov/EM/SPM/Pages/Step1.aspx. Accessed 4 June 2013.

Walsh, C., \& Thornley, P. (2012). Barriers to improving energy efficiency within the process industries with a focus on low grade heat utilisation. Journal of Cleaner Production, 23(1), 138-146.

Venmans, F. (2014). Triggers and barriers to energy efficiency measures in the ceramic, cement and lime sectors. Journal of Cleaner Production, 69, 133-142.

Worrell, E., Laitner, J. A., Ruth, M., \& Finman, H. (2003). Productivity benefits of industrial energy efficiency measures. Energy, 28(11), 1081-1098. 